\title{
Synthesis of Single-Walled Carbon Nanotubes by Induction Thermal Plasma
}

\author{
Keun Su Kim ${ }^{1}$, Ala Moradian², Javad Mostaghimi ${ }^{2}$, Yasaman Alinejad $^{1}$, Ali Shahverdi ${ }^{1}$, Benoit Simard ${ }^{3}$, \\ and Gervais Soucy ${ }^{1}(\bowtie)$ \\ ${ }^{1}$ Department of Chemical Engineering, Université de Sherbrooke, J1K 2R1, Canada \\ ${ }^{2}$ Department of Mechanical and Industrial Engineering, University of Toronto, M5S 3G8, Canada \\ ${ }^{3}$ Steacie Institute for Molecular Sciences, National Research Council, K1A 0R6, Canada \\ Received: 1 May 2009 / Revised: 9 July 2009 / Accepted: 31 August 2009 \\ (C) Tsinghua University Press and Springer-Verlag 2009. This article is published with open access at Springerlink.com
}

\begin{abstract}
The production of high quality single-walled carbon nanotubes (SWCNTs) on a bulk scale has been an issue of considerable interest. Recently, it has been demonstrated that high quality SWCNTs can be continuously synthesized on large scale by using induction thermal plasma technology. In this process, the high energy density of the thermal plasma is employed to generate dense vapor-phase precursors for the synthesis of SWCNTs. With the current reactor system, a carbon soot product which contains approximately $40 \mathrm{wt} \%$ of SWCNTs can be continuously synthesized at the high production rate of $\sim 100 \mathrm{~g} / \mathrm{h}$. In this article, our recent research efforts to achieve major advances in this technology are presented. Firstly, the processing parameters involved are examined systematically in order to evaluate their individual influences on the SWCNT synthesis. Based on these results, the appropriate operating conditions of the induction thermal plasma process for an effective synthesis of SWCNTs are discussed. A characterization study has also been performed on the SWCNTs produced under the optimum processing conditions. Finally, a mathematical model of the process currently under development is described. The model will help us to better understand the synthesis of SWCNTs in the induction plasma process.
\end{abstract}

\section{KEYWORDS}

Single-walled carbon nanotubes (SWCNTs), large-scale continuous synthesis, radio frequency (RF) induction thermal plasma, optimization, numerical modeling

Over the past decade, the superlative properties of single-walled carbon nanotubes (SWCNTs) [1] have attracted much attention for their use in a wide range of applications, including microelectronic [2], biomedical [3], polymer [4], energy [5], and environmental [6] devices. Consequently, the economical production of high quality SWCNTs on a large scale has become an issue of considerable interest. To date, there have been tremendous efforts to develop new synthesis methods leading to the economical production of SWCNTs and it has been shown that SWCNTs can be produced by many

Address correspondence to Gervais.Soucy@USherbrooke.ca 
different ways, such as arc discharge, laser ablation, arc-jet plasmas, chemical vapor deposition (CVD), non-equilibrium plasmas, and combustion methods.

Recently, it was demonstrated that high quality SWCNTs can be continuously synthesized, on a large scale, by the method of simultaneous direct evaporation of carbon black and metal/metaloxide catalyst mixtures, using induction thermal plasma technology [7]. In this process, the high energy density of the thermal plasma is employed to generate dense vapor-phase precursors for the synthesis of SWCNTs. The main advantages of this process are that the rate of input carbon is not limited by the rate of electrode erosion and that SWCNTs are synthesized in the free space without any substrates. Before undertaking optimization efforts, it was shown that a carbon soot product which contains approximately $40 \mathrm{wt} \%$ of SWCNTs can be continuously synthesized at production rates of over $100 \mathrm{~g} / \mathrm{h}$.

The primary goal of this article is to review our recent research advances in this new technology. The review begins with a brief introduction of induction thermal plasma and the processing system developed for the SWCNT synthesis, which is followed by a parametric study of the various variables involved in the process. The characterization of the raw and purified materials is presented in section 1. Section 2 is devoted to our current numerical work on the modeling of the growth of SWCNT in the induction plasma reactor. Preliminary results of this numerical work are presented and comparisons with related experimental data are carried out for validation purposes. Finally, the article is concluded with a discussion of future work.

\section{SWCNT synthesis by induction thermal plasma technology}

\subsection{Induction thermal plasma}

In recent years, thermal plasma jets generated by various plasma torches have begun to be used for the production of various functional nanomaterials by taking advantages of their high temperature and high enthalpy, which have proved to be critical in obtaining high quality products $[8,9]$. Among the variety of thermal plasmas, induction thermal plasma has proven to offer many advantages in designing the nanostructure of the products and seems to be one of the most promising methods for the largescale synthesis of new nanomaterials [10].

Induction thermal plasmas are electrodeless discharges, in which the radio frequency (RF) electrical energy in a copper coil is coupled to a plasma gas by eddy currents through magnetic induction [11, 12]. The excitation frequency is typically between $200 \mathrm{kHz}$ and $40 \mathrm{MHz}$ and laboratory units are operated at power levels of 30$50 \mathrm{~kW}$ while larger scale industrial units have been tested to a power level of $1 \mathrm{MW}$.

A typical induction plasma torch usually consists of three main elements: an induction coil, a confinement tube, and an injection probe. The torch is operated using three distinct gas streams: a feedstock carrier gas, a protective sheath, and a central stream where the chemical transformations take place. The central gas is injected with a swirl component to stabilize the plasma, whereas the sheath gas is injected with a directional flow and is used to protect the torch wall from damage by the high temperature of the discharge. The feedstock materials are introduced axially through a water-cooled probe located at the center of the plasma torch. This injection scheme allows for the delivery of the raw materials directly into the core region of the plasma discharge. In comparison with other types of thermal plasmas, the induction thermal plasma has the following unique advantages in material synthesis: (1) a relatively large plasma volume and a lower plasma velocity compared to those in DC or AC plasmas, thus providing for longer residence times for feedstock materials inside the discharge zone; (2) the RF plasma torch is an almost maintenance-free device because of its electrodeless design; (3) it allows the use of practically any type of plasma gases and feedstock materials without affecting the stability of the discharge.

\subsection{The induction thermal plasma processing system for SWCNT synthesis}

Figure 1 shows a prototype reactor system 
constructed to facilitate the continuous production of SWCNTs on a large scale. The reactor system consists of three major parts: a reaction chamber, a quenching chamber, and a filtration chamber. The induction plasma torch is a commercial TEKNA PL-50 (Tekna Plasma Systems, Inc.) composed of a five-turn coil and a ceramic tube with an internal diameter of 50 $\mathrm{mm}$. The torch is driven by a $40 \mathrm{~kW}$ RF power supply (Lepel Co.) operated at an oscillator frequency of 2-5 $\mathrm{MHz}$.

The reaction chamber is a double-walled cylindrical tube of $500 \mathrm{~mm}$ in length and $150 \mathrm{~mm}$ in inner diameter. The primary purpose of this reaction chamber is to provide a suitable environment for the formation of SWCNT precursors and SWCNT growth. The reaction chamber contains interchangeable graphite inserts to allow for flexible control over maximum achievable temperature, background temperature, and cooling rate. The quenching chamber is composed of a double-walled cylindrical segment of $500 \mathrm{~mm}$ in length, and $150 \mathrm{~mm}$ in inner diameter. The main roles of the quenching system are to provide a region for the completion of the SWCNT growth process and for the initiation of secondary chemical reactions, such as in situ purification or functionalization. For this purpose, the quenching chamber is equipped with multiple ports to allow for the injection of various chemical agents. The filtration

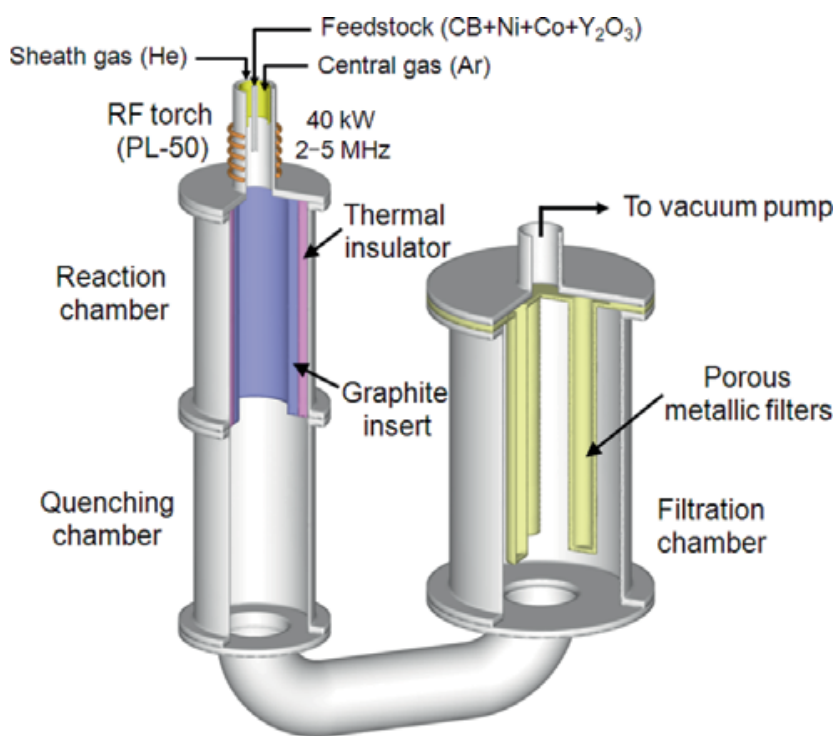

Figure 1 Schematic layout of an induction thermal plasma processing system developed for the continuous production of SWCNTs on large scales chamber consists of three porous metallic filters $(60$ $\mathrm{mm}$ in outer diameter, $850 \mathrm{~mm}$ in length, and with pore size of $2.8 \mu \mathrm{m}$ ) which effectively separate the solid SWCNT-containing materials from the gaseous products. The residual gases are further cooled before being exhausted through a vacuum pump.

The SWCNT synthesis experiments are typically performed at a fixed plate power of $40 \mathrm{~kW}$ and at a constant pressure of $66 \mathrm{kPa}$, the operating frequency being around $3 \mathrm{MHz}$. For stable operation of the induction plasma torch, the sheath gas (He) is maintained at a constant flow rate of $120 \mathrm{slpm}$ (slpm denotes standard liters per minute), while the central gas (Ar) is supplied at a flow rate of $25 \mathrm{slpm}$. The powder carrier gas (Ar) is introduced through the injection probe at a flow rate of 5 slpm. Lastly, powders are delivered at feed rates typically between 72 and $120 \mathrm{~g} / \mathrm{h}$. Under these operating conditions, the torch thermal efficiency is around $65 \%$.

\subsection{Parametric study of processing conditions}

Changes in the processing parameters, such as plasma gas composition and velocities, operating pressures, composition of raw materials, and feed rates usually bring about important modifications of the synthesis environment and consequently have a significant influence on the final yield of SWCNTs. In an effort to optimize the induction thermal plasma process for the SWCNT production, a parametric study of the various processing parameters has been conducted systematically.

Due to their different nucleation temperatures, solubilities of carbon, and catalytic activities for graphitization, the catalysts play a critical role in determining the type, properties, and yield of SWCNTs [13]. Thus, the effect of varying the catalytic source was first analyzed. Since many studies have reported that bimetallic catalysts such as $\mathrm{Ni} / \mathrm{Y}$ or $\mathrm{Ni} / \mathrm{Co}$ enhance the yield of SWCNTs compared to single-metal catalyst systems [14], it was decided to investigate possible synergistic effects within three catalyst mixtures. Experiments were performed by adding $\mathrm{Y}_{2} \mathrm{O}_{3}$ or $\mathrm{CeO}_{2}$ to a previously employed bimetallic catalyst (a mixture of $\mathrm{Ni}$ and $\mathrm{Co}$ ). Figure 2(a) shows a comparison of the Raman spectra of the resulting materials. The material produced using a 
mixture of $\mathrm{Ni} / \mathrm{Co} / \mathrm{Y}_{2} \mathrm{O}_{3}$ exhibits the highest G-band (G-graphite) to D-band (D-disorder) intensity ratio, which indicates a much better graphitization of SWCNTs in the as-produced soot.

The positive impact on SWCNT production of the addition of $\mathrm{Y}_{2} \mathrm{O}_{3}$ or $\mathrm{CeO}_{2}$ was explained in our previous work in terms of the following contributing factors [7]: (1) the oxygen released upon their vaporization can help burn away amorphous carbon during the process; (2) it is very likely that the addition of $\mathrm{Y}$ or Ce significantly increases radiative emissions from the plasma due to their low ionization potentials and, consequently, radiative heat transfer, and possibly photocatalytic reactions, will be promoted; (3) the presence of $Y$ in the metal particle may alter the process temperature through the highly exothermic reaction of yttrium carbide formation which would have the effect of retarding the start of SWCNT growth and maintaining the particles in a liquid phase, allowing for a higher carbon supersaturation than for pure Ni or Co particles.

At high temperatures, the thermodynamic properties of plasma gases depend strongly on their compositions [15]. Thus, an experiment was performed using a mixture of $\mathrm{He}$ and $\mathrm{Ar}$ as the sheath gas (He:Ar=1:1), in order to clarify the effect of sheath gas composition on the quality of SWCNTs produced. The normalized Raman spectra obtained with $\mathrm{He}$ and the $\mathrm{He} / \mathrm{Ar}$ mixture as sheath gas are shown in Fig. 2(b). From the increased intensity of the D-band, the addition of Ar into the sheath gas seems to have a negative impact on SWCNT quality. The high efficiency of the He sheath gas can be

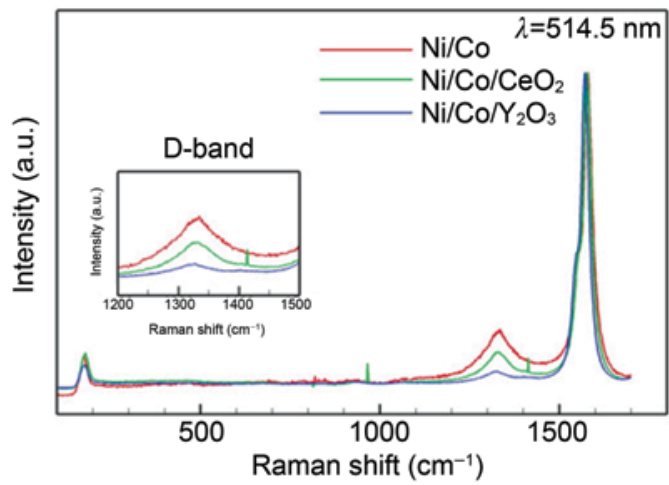

(a) explained by its good thermal conductivity which results in the faster and more intense evaporation of the feedstock in the He gas. It was also found from the previous simulation results that higher quenching rates are obtainable in He plasmas through efficient heat exchange with the surroundings [7]. This is very attractive for the rapid generation of SWCNT precursors by the strong supersaturation of the carbon vapors produced. Thus, gas compositions with higher He contents seem to be more efficient for the synthesis. However, the stability of the plasma typically decreases on replacement of Ar with He, due to the lower electrical conductivity and higher ionization potential of the latter.

It is well known that the physico-chemical properties of the solid carbon sources, such as particle size, sulfur content, and grain size, all have significant effects on the quality of the SWCNTs produced [16]. For this reason, four different grades of carbon black (Super P, Raven 860 ultra, Elftex-12, and M-880) were tested and their performances were evaluated by analyzing the Raman spectra of the products. In Table 1 , some important properties of these carbon blacks are summarized. Sulfur-containing compounds have been routinely employed to improve SWCNT yields in other synthesis processes. It is believed that small amounts of sulfur can help promote the SWCNT growth reaction by changing the surface tension and thus affecting reaction paths, while higher sulfur concentrations may poison the catalyst particles and impede the formation of SWCNTs [17]. In Fig. 3(a), the three carbon blacks have different sulfur contents while having very similar sizes of primary

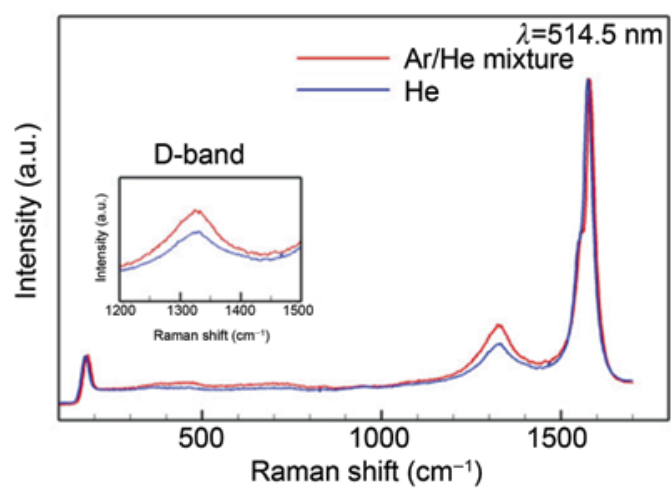

(b)

Figure 2 Raman spectra of the SWCNTs produced by the induction thermal plasma process showing the effects of (a) different catalysts and (b) sheath gas composition 


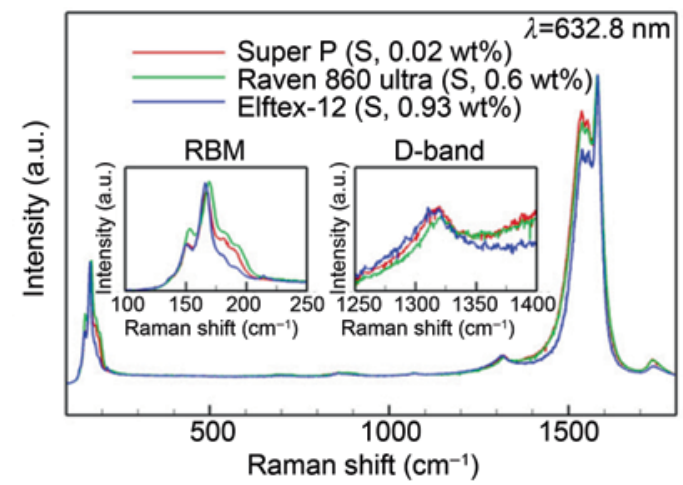

(a)

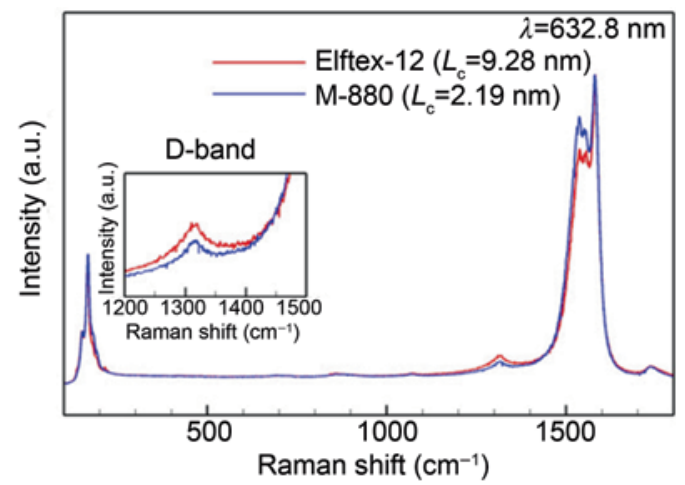

(a)

Figure 3 Raman spectra of the SWCNTs produced by the induction thermal plasma process showing the effect of different grades of carbon black: (a) effect of sulfur content; (b) effect of crystallite size

Table 1 Material properties of the carbon blacks employed for the production of SWCNTs by the induction thermal plasma process

\begin{tabular}{lllll}
\hline Carbon black & Size of primary particle $(\mathrm{nm})$ & Sulfur content $(\mathrm{wt} \%)$ & $d_{002}(\AA)^{\mathrm{a}}$ & $L_{\mathrm{c}}(\mathrm{nm})^{\mathrm{a}}$ \\
\hline Super P & 40 & 0.02 & 3.575 & 7.908 \\
Raven 860 ultra & 39 & 0.60 & 3.601 & 8.290 \\
Elftex-12 & 37 & 0.93 & 3.645 & 9.277 \\
M-880 & 16 & 1.11 & 3.578 & 2.187 \\
\hline
\end{tabular}

${ }^{a} d_{002}$ is the interplanar distance between two graphene layers and $L_{c}$ is the crystallite size.

particles and grains. Our results agree with previous observations that sulfur content has an effect on the diameter distribution of SWCNTs [7, 17]; larger tubes are synthesized with higher levels of sulfur (see RBM in Fig. 3(a)).

The influence of the microstructure of carbon blacks on SWCNT growth was also investigated. By using powder X-ray diffraction (XRD) patterns and the Debye-Scherrer formula, the crystallite sizes of the individual carbon blacks $\left(L_{c}\right)$ were calculated and are presented in Table 1. Carbon black M-880 $\left(L_{\mathrm{c}}=\right.$ $2.187 \mathrm{~nm}$ ) exhibits the lowest degree of graphitization among the four carbon blacks and outperforms Elftex-12, which has the largest grain size of $9.277 \mathrm{~nm}$ (see Fig. 3(b)). These results indicate that the efficiency of carbon blacks for the production of SWCNTs decreases with their crystallite size. This is because the smaller, less-ordered grains require less energy for their complete vaporization within the plasma.

\subsection{Characterization of the SWCNTs produced}

By using the optimum processing conditions found in the above parametric study (i.e., Raven 860 ultra/ $\mathrm{Ni} / \mathrm{Co} / \mathrm{Y}_{2} \mathrm{O}_{3}$ with $98.4 / 0.6 / 0.6 / 0.4$ at\%), SWCNTs were produced and their material properties were analyzed. Under these conditions, large rubbery textured sheets of SWCNT material were formed in the filtration chamber after only 15-20 min of operation (Fig. 4 (a)). To determine what types of materials were synthesized during the process, the raw soot material was examined using powder XRD and thermogravimetric analysis (TGA). The XRD pattern shows a rich crystalline structure, as seen in Fig. 4(b). Interestingly, the presence of yttrium carbide $\left(\mathrm{YC}_{2}\right)$ is readily observable but there are no traces of nickel or cobalt carbides (e.g., $\mathrm{Ni}_{3} \mathrm{C}, \mathrm{CO}_{2} \mathrm{C}$, and $\mathrm{Co}_{3} \mathrm{C}$ ). Although the background is high at small $2 \theta$, no peak attributable to fullerenes is visible in the $2 \theta$ range $10^{\circ}-20^{\circ}$.

Figure 4(c) shows the percentage weight loss and its derivative as a function of temperature for the raw soot material in air. The majority of the carbonaceous materials are consumed within multiple oxidation events occurring between 300 and $650{ }^{\circ} \mathrm{C}$. The residual mass of $16.2 \mathrm{wt} \%$, observed in the TGA trace, corresponds to the fully oxidized catalyst metals. The derivative curve (DTG) for the soot material was fitted to a series of Lorentzian functions and exhibited six peaks at $312(14 \%), 342(28 \%), 398$ (19\%), $440(8 \%), 575(11 \%)$, and $600{ }^{\circ} \mathrm{C}(20 \%)$ due to 
the combustion of various forms of carbonaceous materials. Although the interpretation of the peaks observed in DTG profiles can be very challenging, the individual oxidation events in the DTG profile were tentatively correlated to specific sample components as shown in Fig. 4(c). The DTG profile exhibits a strong peak in the higher temperature range of 600 $-750{ }^{\circ} \mathrm{C}$ (i.e., the burning temperature of partially graphitized carbon particles). In our previous study
[18], this was mainly attributed to unprocessed starting feedstock materials. While we still believe this is plausible, it could also originate from highly graphitized reaction by-products such as multi-layer carbon shell particles.

Further characterization by Raman spectroscopy, scanning electron microscopy (SEM), and transmission electron microscopy (TEM) were performed, and the results are shown in Figs. 5(a)-(d).

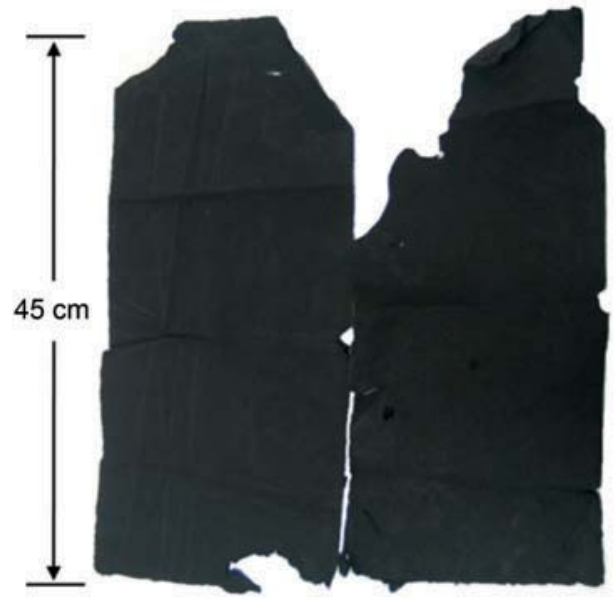

(a)

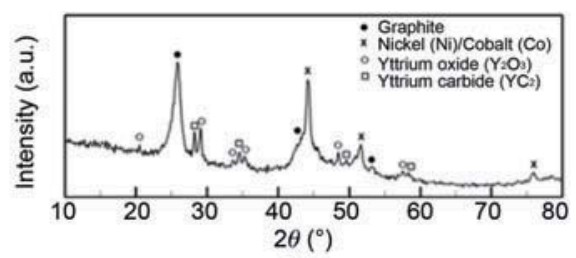

(b)

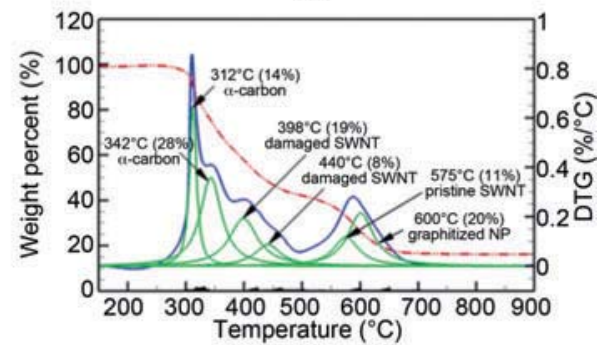

(c)

Figure 4 (a) Photograph; (b) XRD pattern; (c) TGA/DTG plots of the raw SWCNT soot produced by the induction thermal plasma process

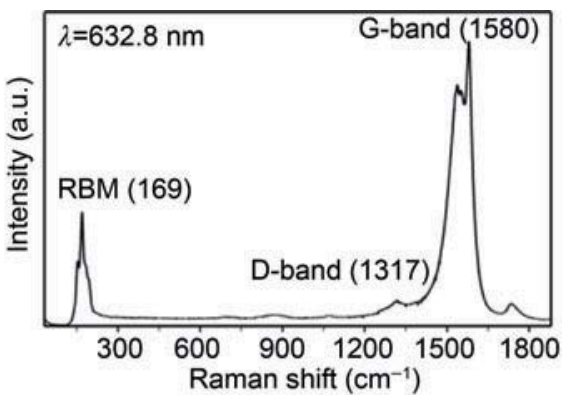

(a)

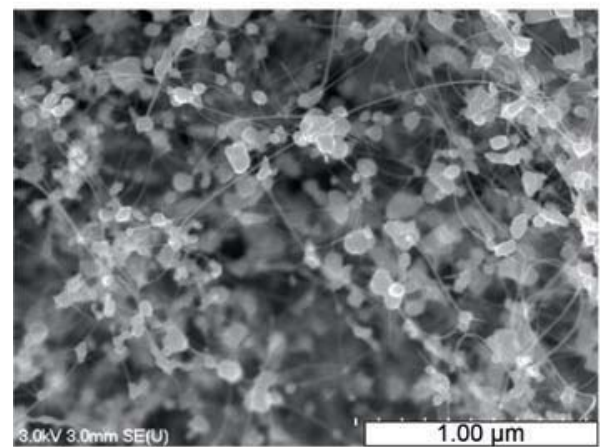

(c)

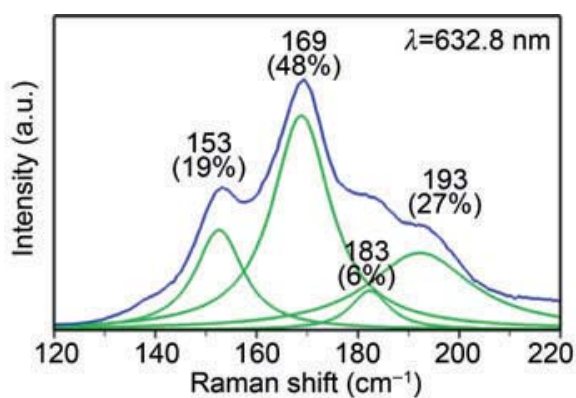

(b)

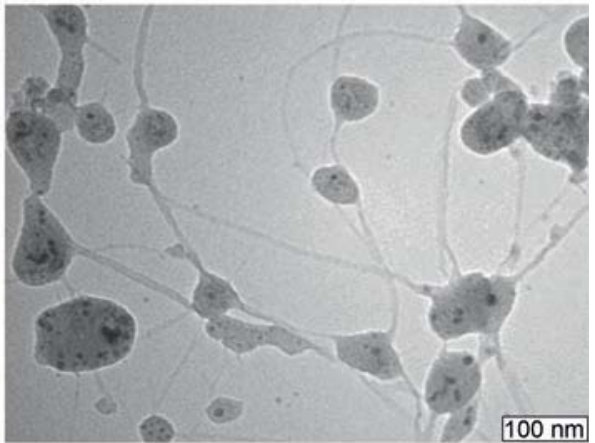

(d)

Figure 5 (a) Full Raman spectrum; (b) low-frequency region of the Raman spectrum; (c) SEM image; (d) TEM image of the raw SWCNT soot produced by the induction thermal plasma process 

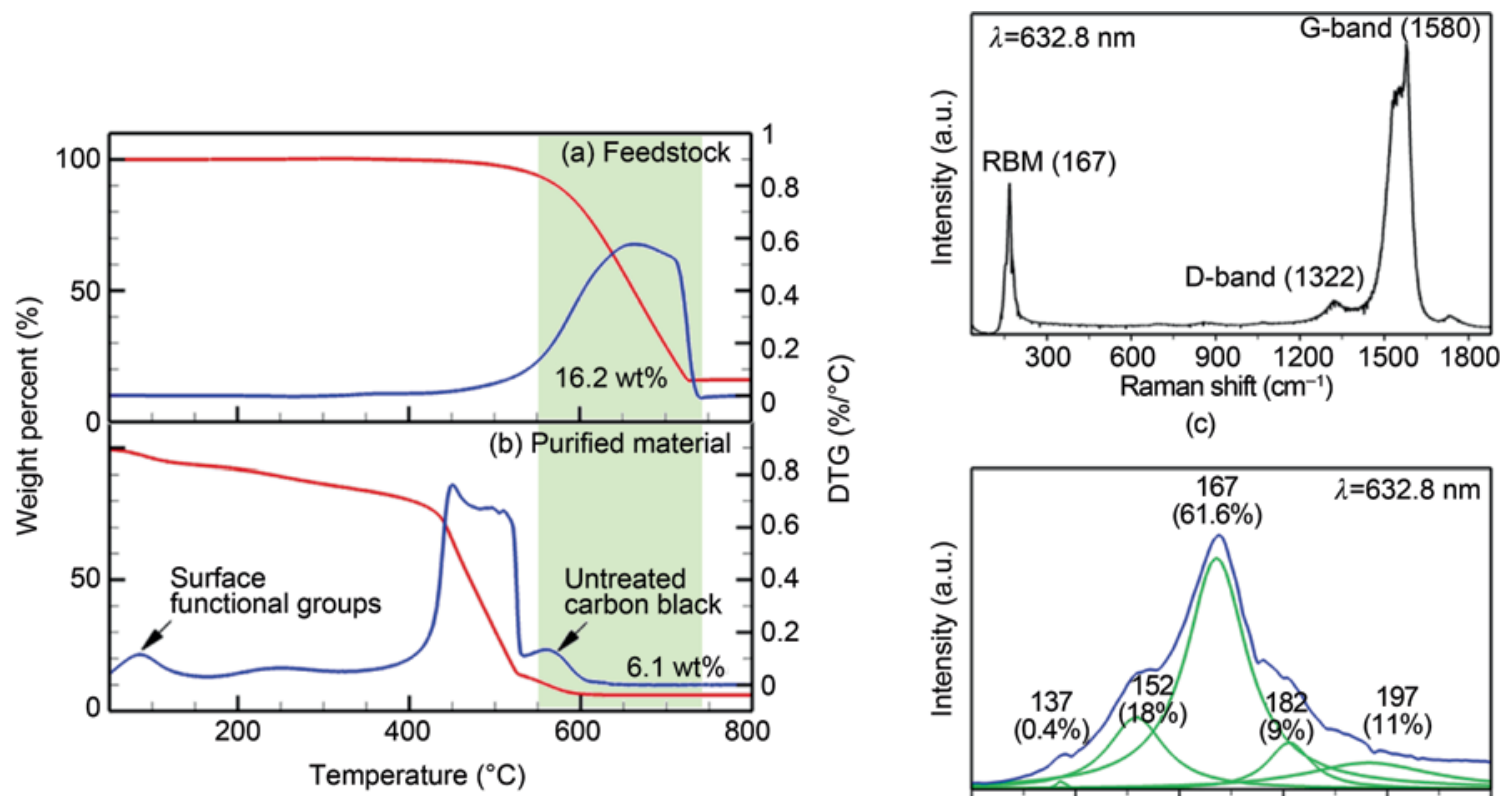

(c)

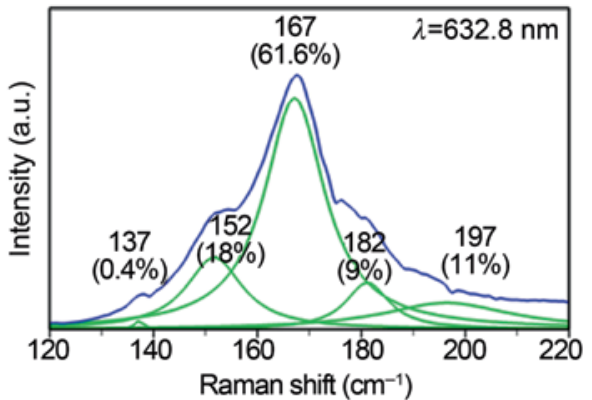

(d)

Figure 6 (a) TGA/DTG plots of the feedstock; (b) TGA/DTG plots of the purified SWCNT film; (c) full Raman spectrum of the purified SWCNT film; (d) low-frequency region of the Raman spectrum of the purified SWCNT film

From the expanded view of the radial breathing mode (RBM) in the Raman spectrum (Fig. 5(b)), the diameters of our tubes seem to be in the range of 1.2 $-1.6 \mathrm{~nm}$, with the majority being $1.4 \mathrm{~nm}$ according to the equation $\omega=12.5+223.5 / d$ [19], where $d$ is the tube diameter, in $\mathrm{nm}$, and $\omega$ is the wave number, in $\mathrm{cm}^{-1}$. The mean diameter is larger than that of HipCo SWCNT materials and very similar to those obtained from materials produced by laser ablation or arc discharge.

Figures 6(a) and (b) show the TGA/DTG profiles of the feedstock and of the purified SWCNTs, respectively. The purified SWCNTs exhibit a welldefined one-step weight loss between 400 and $550{ }^{\circ} \mathrm{C}$, as seen in Fig. 6(b). But a very weak peak at a temperature of around $625{ }^{\circ} \mathrm{C}$ is also visible in Fig. 6(b), which overlaps with the oxidation peak of the untreated carbon black (Fig. 6(a)). It is apparent from the Raman spectra in Figs. 6(c) and 6(d) that the purification process narrows the diameter distribution of the SWCNTs produced, by preferential removal of the smaller diameter tubes.

The BET surface areas of the raw soot and of the purified SWCNTs were derived from the $\mathrm{N}_{2}$ adsorption isotherms at $77 \mathrm{~K}$ to be 122 and $480 \mathrm{~m}^{2} / \mathrm{g}$, respectively. The BET surface area of the purified SWCNTs measured in this work is in good agreement with those of purified arc discharge-grown SWCNTs $\left(\sim 400 \mathrm{~m}^{2} / \mathrm{g}\right)$, which have somewhat similar tube diameter distributions [20]. The electrical resistivities of the samples were measured by using the fourprobe method and the results are available in the literature [18].

\section{Numerical modeling of the SWCNT growth in the induction plasma process}

Although the initial configurations of the process were designed largely through empirical approaches, the optimization procedure requires a more complete understanding of the SWCNT formation mechanism and its relationship with the local plasma properties (which can be controlled by the macroscopic processing parameters) [21-23]. However, our knowledge of the plasma characteristics during the SWCNT synthesis is rather limited so far, the plasma zone being frequently considered as a black box. The reason for this is mainly due to limitations on the accessibility of diagnostic tools, attributable to the high heat flux, and the fast fluctuation of the plasma 
resulting from the chaotic interactions with the feedstock materials injected.

Since information about the processing conditions - including the plasma propertiesis crucial to further advance our current induction plasma technology, a mathematical model based on the widely accepted vapor-liquid-solid (VLS) growth mechanism has been developed [24]. The goal of this numerical work is to provide a selfconsistent description of the plasma characteristics for given operating conditions, and to then accurately predict the yield rate of the SWCNTs. For this, the mathematical model developed consists of several sub-models: (1) plasma generation model; (2) plasmaparticle interaction model; (3) carbon-metal liquid droplet formation model; (4) SWCNT nucleation and growth model. A more detailed explanation of each model follows in this section.

\subsection{Plasma generation model}

To simulate plasma generation by an RF induction plasma torch, a 2-D plasma model has been developed based on the following assumptions [25]: (1) an axisymmetric and steady-state; (2) a quasi-neutral and optically thin plasma in a local thermodynamic equilibrium (LTE) state; (3) negligible viscous dissipation and pressure work terms in the energy equation; (4) incompressible and temperature-dependent mass density; (5) a negligible effect of metal vapor on the electrical conductivity of the plasma; (6) a negligible effect of carbon and metal vapors on the radiation property of the plasma; (7) turbulent flow. Although the vapors generated from the evaporation of particles have a significant effect on the electrical and radiational properties of the plasma, their effects were not taken into account because no reliable data are available. Based on these assumptions, the coupled interactions between eddy current, induced magnetic field, and plasma flow can be described in the framework of magnetohydrodynamic (MHD) equations for conservation of mass, momentum, and energy, along with the $K$ $-\varepsilon$ turbulence model. The axisymmetric cylindrical coordinate system $(r, z)$ was adopted in this modeling work and detailed governing equations for the plasma generation model are as follows:
Mass conservation:

$$
\nabla \cdot(\rho \boldsymbol{u})=S_{\mathrm{p}}^{\mathrm{c}} .
$$

Momentum conservation:

$$
\nabla \cdot(\rho \boldsymbol{u u})=-\nabla p+\nabla \cdot \tau+F_{\mathrm{L}}+S_{\mathrm{p}}^{\mathrm{m}} .
$$

Energy conservation:

$$
\nabla \cdot(\rho \boldsymbol{u} h)=\nabla \cdot\left(\frac{\boldsymbol{x}_{\mathrm{eff}}}{C_{\mathrm{p}}} \nabla h\right)+P_{\mathrm{ohm}}-R_{\mathrm{rad}}+S_{\mathrm{p}}^{\mathrm{e}}
$$

Turbulent kinetic energy:

$$
\nabla \cdot(\rho \boldsymbol{u} K)=\nabla \cdot\left[\left(\mu+\frac{\mu_{\mathrm{t}}}{P r_{K}}\right) \nabla K\right]+G_{\mathrm{t}}-\rho \varepsilon .
$$

Dissipation rate of turbulent kinetic energy:

$$
\nabla \cdot(\rho \boldsymbol{u} \varepsilon)=\nabla \cdot\left[\left(\mu+\frac{\mu_{\mathrm{t}}}{P r_{\varepsilon}}\right) \nabla \varepsilon\right]+\frac{\varepsilon}{K}\left(C_{1} G_{\mathrm{t}}-C_{2} \rho \varepsilon\right) .
$$

Species conservation $(\mathrm{Ar}, \mathrm{He}, \mathrm{Ni}, \mathrm{Co}$, and $\mathrm{C})$ :

$$
\nabla \cdot\left(\rho \boldsymbol{u} Y_{\mathrm{k}}\right)=\nabla \cdot\left(\left(\rho D_{k}+\frac{\mu_{\mathrm{t}}}{S c_{\mathrm{t}}}\right)_{\nabla} Y_{k}\right)+S_{\mathrm{p}}^{\mathrm{k}}
$$

In the $K-\varepsilon$ model, the effective viscosity and thermal conductivity include both laminar and turbulent components,

$$
\mu_{\text {eff }}=\mu+\mu_{\mathrm{t}} \text { and } x_{\text {eff }}=x+x_{\mathrm{t}} .
$$

In the above equations, the turbulent viscosity $\mu_{t}$ and the turbulent thermal conductivity $\varkappa_{t}$ are defined as

$$
\mu_{\mathrm{t}}=\rho C_{\mu} \frac{K^{2}}{\varepsilon} \text { and } x_{\mathrm{t}}=\frac{\mu_{\mathrm{t}} C_{\mathrm{p}}}{P r_{\mathrm{t}}} .
$$

For the Lorentz force and ohmic heating terms appearing in the momentum and energy conservation equations, it is necessary to calculate the electromagnetic field distributions inside the plasma torch, induced by the alternating coil current. The electromagnetic field distribution can be obtained by solving the magnetic vector potential equation as described in Refs. [25, 26].

Magnetic vector potential:

$$
\frac{1}{r} \frac{\partial}{\partial r}\left(r \frac{\partial A_{\theta}}{\partial r}\right)+\frac{\partial^{2} A_{\theta}}{\partial z^{2}}-\frac{A_{\theta}}{r^{2}}=-\mu_{0}\left(J_{\text {coil }}+J_{\text {ind }}\right) .
$$

Electric and magnetic fields:

$$
E_{\theta}=-\mathrm{i} 2 \pi f A_{\theta}, \quad \mu_{0} H_{\mathrm{z}}=\frac{1}{r} \frac{\partial\left(r A_{\theta}\right)}{\partial r}, \quad \mu_{0} H_{\mathrm{r}}=-\frac{\partial A_{\theta}}{\partial z} .
$$

Lorentz forces and ohmic heating: 


$$
\begin{aligned}
& F_{\mathrm{r}}=\frac{1}{2} \mu_{0} \sigma \operatorname{Re}\left[E_{\theta} H_{\mathrm{z}}^{*}\right], F_{\mathrm{z}}=-\frac{1}{2} \mu_{0} \sigma \operatorname{Re}\left[E_{\theta} H_{\mathrm{r}}^{*}\right], \\
& P_{\mathrm{ohm}}=\frac{1}{2} \sigma \operatorname{Re}\left[E_{\theta} E_{\theta}^{*}\right] .
\end{aligned}
$$

In the above equations, the superscript * denotes the complex conjugate.

\subsection{Plasma-particle interaction model}

In the induction thermal plasma process, SWCNTs are continuously synthesized from direct evaporation of carbon-containing particles inside the plasma jet, and consequently the SWCNTs yield rate strongly depends on the evaporation efficiency of the particle injected. In spite of this importance, particle behaviors in the thermal plasmas are not yet totally understood. Therefore, the following simplifications were assumed in this work [27]: (1) spherical particles were assumed; (2) a uniform temperature distribution inside a particle was assumed; (3) particle sizes were assumed to follow the Rosin-Rammler distribution function; (4) the temperature variation in the particle boundary layer and the rarefaction effect (Knudsen effect) were not taken into account [28].

The Lagrangian reference frame approach was employed to describe particle behaviors inside the plasma plume $[27,29]$. In this method, the momentum exchange between particles and plasma are calculated by Newton's law with a proper drag coefficient (i.e., the force balance for the equation of motion is restricted to the viscous drag and inertia), whereas the heat and mass transfers are calculated by using the energy balance equations as described in Refs. [27, 29].

Momentum exchange between plasma and particle:

$$
\begin{aligned}
& m_{\mathrm{p}} \frac{\mathrm{d} \boldsymbol{u}_{\mathrm{p}}}{\mathrm{d} t}=\frac{\pi}{8} d_{\mathrm{p}}^{2} \rho C_{\mathrm{D}}\left(\boldsymbol{u}-\boldsymbol{u}_{\mathrm{p}}\right)\left|\boldsymbol{u}-\boldsymbol{u}_{\mathrm{p}}\right|, \\
& C_{\mathrm{D}}=\left(24 / R e_{\mathrm{p}}\right) f\left(R e_{\mathrm{p}}\right), \quad R e_{\mathrm{p}}=\frac{\rho d_{\mathrm{p}}\left|\boldsymbol{u}-\boldsymbol{u}_{\mathrm{p}}\right|}{\mu},
\end{aligned}
$$

where $C_{D}$ is the drag coefficient proposed in Ref. [30] and the integration time $t$ is until the diameter of the particle becomes zero (i.e., complete evaporation).

Heat and mass exchanges between plasma and particle:

$$
Q=\pi d_{\mathrm{p}}^{2} h_{\mathrm{c}}\left(T-T_{\mathrm{p}}\right)-\pi d_{\mathrm{p}}^{2} \varepsilon_{\mathrm{p}} \sigma_{\mathrm{sb}}\left(T_{\mathrm{p}}^{4}-T_{\mathrm{a}}^{4}\right) .
$$

The heat transfer coefficient $\left(h_{c}\right)$ is given with the Nusselt number by using the correlation of Ranz and Marshall [31, 32].

$$
h_{\mathrm{c}}=\frac{\varkappa \cdot N u}{d_{\mathrm{p}}}, N u=2.0+0.6 \operatorname{Re}^{0.5} \operatorname{Pr}^{0.33}
$$

\subsection{Carbon-metal liquid droplet formation model}

The carbon and metal vapors produced in the plasma plume will be transported to the lower-temperature region, through diffusion or convection processes. According to the VLS growth mechanism, these vapors are cooled down and form a carbon-metal liquid droplet. Although the formation mechanism of carbon-metal droplets is not yet clear, it is likely that carbon vapors first condense into clusters and then metal vapors convert to a liquid droplet by absorbing the carbon clusters produced. In this work, however, it is assumed that a carbon-metal liquid droplet is simply formed by the homogeneous nucleation of metal vapors and subsequently grows through the co-condensation of carbon and metal vapors (i.e., as soon as stable metallic nanoparticles appear by homogeneous nucleation, carbon atoms, and the rest of the not-yet-nucleated metal atoms condense on the surface of the nanoparticles produced) and coagulation proceeds. Thus, the clustering of carbon atoms and formation of soot nuclei were not taken into account due to many uncertainties associated with these phenomena.

With this simplified nucleation and growth pathways, the droplet generation process can be described by the aerosol general dynamic equation (GDE) [33]. For this, the following assumptions are further introduced in this work [29]: (1) spherical droplets; (2) negligible droplet inertia; (3) identical velocity and temperature of a droplet to those of the gas flow; (4) negligible heat generation caused by condensation; (5) negligible electric charge of a droplet; (6) lognormal size distribution; (7) the composition of a droplet at a local position is independent on the droplet size; (8) the effect of SWCNTs grown from a droplet is totally ignored, even though SWCNTs have a significant impact on the droplet generation and its transport. The GDE can be written as 


$$
\begin{gathered}
\frac{\partial n\left(v_{\mathrm{d}}\right)}{\partial t}=-\nabla \cdot\left(\boldsymbol{u} n\left(v_{\mathrm{d}}\right)\right)-\nabla \cdot\left(\boldsymbol{u}_{\mathrm{th}} n\left(v_{\mathrm{d}}\right)\right)+\nabla \cdot\left(D_{\mathrm{d}}\left(v_{\mathrm{d}}\right) \nabla n\left(v_{\mathrm{d}}\right)\right) \\
+I \cdot \delta\left(v_{\mathrm{d}}-v_{\mathrm{d}}^{*}\right)-\frac{\partial\left[G n\left(v_{\mathrm{d}}\right)\right]}{\partial v_{\mathrm{d}}} \\
+\frac{1}{2} \int_{0}^{v_{\mathrm{d}}} \beta\left(v_{\mathrm{d}}^{\prime}, v_{\mathrm{d}}-v_{\mathrm{d}}^{\prime}\right) n\left(v_{\mathrm{d}}^{\prime}\right) n\left(v_{\mathrm{d}}-v_{\mathrm{d}}^{\prime}\right) \mathrm{d} v_{\mathrm{d}}^{\prime} \\
-\int_{0}^{\infty} \beta\left(v_{\mathrm{d}}, v_{\mathrm{d}}^{\prime}\right) n\left(v_{\mathrm{d}}\right) n\left(v_{\mathrm{d}}^{\prime}\right) \mathrm{d} v_{\mathrm{d}}^{\prime}
\end{gathered}
$$

where the first, second, and third terms describe mass transfer inside a control volume caused by convection, thermophoresis, and diffusion processes, respectively. The fourth term is the metal nuclei generation term through the homogeneous nucleation. In this work, these metal nuclei are assumed to form carbon-metal liquid droplets by absorbing both metal and carbon vapors. This process is described by the fifth term, which represents the growth of carbon-metal droplets by the cocondensation process. The sixth and seventh terms stand for the further growth of carbon-metal droplets through the coagulation process.

The thermophoretic velocity $\boldsymbol{u}_{\mathrm{th}}$ is given as

$$
\boldsymbol{u}_{\mathrm{th}}=-0.55 \cdot \frac{\mu}{\rho} \frac{\nabla T}{T},
$$

and the size-dependent diffusion coefficient $D_{\mathrm{d}}$ is written as [34],

$$
D_{\mathrm{d}}\left(v_{\mathrm{d}}\right)=\frac{k_{\mathrm{B}} T}{3 \pi \mu}\left[\left(\frac{\pi}{6 v_{\mathrm{d}}}\right)^{1 / 3}+3.314 \lambda\left(\frac{\pi}{6 v_{\mathrm{d}}}\right)^{2 / 3}\right],
$$

in which the mean free path $\lambda$ is defined as,

$$
\lambda=\frac{\mu}{0.499 p\left(8 M_{\mathrm{g}} / \pi R T\right)^{1 / 2}} .
$$

The homogeneous nucleation term is given by the self-consistent classical theory proposed in Ref. [35],

$$
I=\frac{\beta\left(v_{1}, v_{1}\right) n_{\mathrm{ms}}^{2} S_{\mathrm{m}}}{12} \sqrt{\frac{\Theta}{2 \pi}} \exp \left(\Theta-\frac{4 \Theta^{3}}{27\left(\ln S_{\mathrm{m}}\right)^{2}}\right),
$$

where the supersaturation ratio of metal vapor $S_{\mathrm{m}}$ and the normalized surface tension $\Theta$ are defined as,

$$
S_{\mathrm{m}}=\frac{P_{\mathrm{vap}}^{\mathrm{m}}}{P_{\mathrm{s}}^{\mathrm{m}}}, \quad \Theta=\frac{\sigma_{\mathrm{st}} S_{\mathrm{m} 1}}{k_{\mathrm{B}} T} .
$$

If the volume of a droplet particle is taken as the sum of the volumes of metal and carbon,

$$
v_{\mathrm{d}}=v_{\mathrm{d}}^{\mathrm{m}}+v_{\mathrm{d}}^{\mathrm{c}}
$$

then the co-condensation term can be written as,

$$
\begin{gathered}
G\left(v_{\mathrm{d}}\right)=\frac{\mathrm{d} v_{\mathrm{d}}}{\mathrm{d} t}=\frac{\mathrm{d} v_{\mathrm{d}}^{\mathrm{m}}}{\mathrm{d} t}+\frac{\mathrm{d} v_{\mathrm{d}}^{\mathrm{c}}}{\mathrm{d} t} \\
=\frac{\pi d_{\mathrm{d}}^{2} v_{\mathrm{m} 1}\left(P_{\text {vap }}^{\mathrm{m}}-P_{\mathrm{s}}^{\mathrm{m}}\right)}{\left(2 \pi m_{\mathrm{m} 1} k_{\mathrm{B}} T\right)^{1 / 2}}+\frac{\pi d_{\mathrm{d}}^{2} v_{\mathrm{c} 1}\left(P_{\text {vap }}^{\mathrm{c}}-P_{\mathrm{s}}^{\mathrm{c}}\right)}{\left(2 \pi m_{\mathrm{c} 1} k_{\mathrm{B}} T\right)^{1 / 2}} .
\end{gathered}
$$

Lastly, in a free molecular regime, the collision kernel between droplets with volume $v_{\mathrm{d}}$ and $v_{\mathrm{d}}^{\prime}$ is written as [36],

$\beta\left(v_{\mathrm{d}}, v_{\mathrm{d}}^{\prime}\right)=\left(\frac{3}{4 \pi}\right)^{1 / 6}\left(\frac{6 k_{\mathrm{B}} T}{\rho_{\mathrm{d}}}\right)^{1 / 2}\left(\frac{1}{v_{\mathrm{d}}}+\frac{1}{v_{\mathrm{d}}^{\prime}}\right)^{1 / 2}\left(v_{\mathrm{d}}^{1 / 3}+v_{\mathrm{d}}^{\prime 1 / 3}\right)^{2}$.

It is very difficult to solve the above GDE directly because of its non-linearity. Up to now, several methods have been proposed to solve this equation, for instance, the discrete sectional method, the moment method, and the quadrature moment method. The moment method has been employed in the present work. In this method, the droplet size distribution function is assumed to follow a lognormal distribution,

$$
n\left(v_{\mathrm{d}}\right)=\frac{\mathrm{d} N}{\mathrm{~d} v_{\mathrm{d}}}=\frac{N}{3 \sqrt{2 \pi} \ln \sigma_{\mathrm{g}}} \exp \left[-\frac{\ln ^{2}\left(v_{\mathrm{d}} / v_{\mathrm{g}}\right)}{18 \ln ^{2} \sigma_{\mathrm{g}}}\right] \cdot \frac{1}{v_{\mathrm{d}}},
$$

and then the first three moments are calculated using the following equations,

$$
M_{\mathrm{k}}=\int_{0}^{\infty} v_{\mathrm{d}}^{\mathrm{k}} n\left(v_{\mathrm{d}}\right) \mathrm{d} v_{\mathrm{d}}, \hat{M}_{\mathrm{k}}=\frac{M_{\mathrm{k}}}{\rho n_{0}\left(v_{\mathrm{m} 1}\right)^{\mathrm{k}}} \quad(k=0,1,2) .
$$

The zeroth moment is the mean droplet number density, whereas the first moment represents the mean volume of the droplets produced. By using the moment method, the GDE can be transformed into these three steady-state moment transport equations [36].

$0^{\text {th }}$ moment:

$$
\nabla \cdot\left(\rho \boldsymbol{u} \hat{M}_{0}\right)=\nabla \cdot\left[\left(\rho \bar{D}_{\mathrm{d}}+\frac{\mu_{\mathrm{t}}}{S c_{\mathrm{t}}}\right) \nabla \hat{M}_{0}\right]-\nabla \cdot\left(\rho \boldsymbol{u}_{\mathrm{th}} \hat{M}_{0}\right)+\frac{I}{n_{0}}
$$$$
-b_{0} B_{2} \rho^{2} n_{0}\left(\hat{M}_{2 / 3} \hat{M}_{-1 / 2}+2 \hat{M}_{1 / 3} \hat{M}_{-1 / 6}+\hat{M}_{1 / 6} \hat{M}_{0}\right) \text {. }
$$

$1^{\text {st }}$ moment:

$$
\begin{aligned}
& \nabla \cdot\left(\rho \boldsymbol{u} \hat{M}_{1}\right)=\nabla \cdot\left[\left(\rho \bar{D}_{\mathrm{d}}+\frac{\mu_{\mathrm{t}}}{S \mathcal{C}_{\mathrm{t}}}\right) \nabla \hat{M}_{1}\right]-\nabla \cdot\left(\rho \boldsymbol{u}_{\mathrm{th}} \hat{M}_{1}\right)+\frac{I}{n_{0}} j^{*} \\
& +B_{1}^{\mathrm{m}} \rho\left(S_{\mathrm{m}}-1\right) \hat{M}_{2 / 3}+B_{1}^{\mathrm{c}} \rho\left(S_{\mathrm{c}}-1\right) \hat{M}_{2 / 3} . \\
& \quad 2^{\text {nd }} \text { moment: }
\end{aligned}
$$$$
2^{\text {nd }} \text { moment: }
$$ \\ $2^{\text {nd }}$ moment:}




$$
\begin{aligned}
& \nabla \cdot\left(\rho \boldsymbol{u} \hat{M}_{2}\right)=\nabla \cdot\left[\left(\rho \bar{D}_{\mathrm{d}}+\frac{\mu_{\mathrm{t}}}{S c_{\mathrm{t}}}\right) \nabla \hat{M}_{2}\right]-\nabla \cdot\left(\rho \boldsymbol{u}_{\mathrm{th}} \hat{M}_{2}\right)+\frac{I}{n_{0}}\left(j^{*}\right)^{2} \\
& +2 B_{1}^{\mathrm{m}} \rho\left(S_{\mathrm{m}}-1\right) \hat{M}_{5 / 3}+2 B_{1}^{\mathrm{c}} \rho\left(S_{\mathrm{c}}-1\right) \hat{M}_{5 / 3} \\
& +2 b_{2} B_{2} \rho^{2} n_{0}\left(\hat{M}_{5 / 3} \hat{M}_{1 / 2}+2 \hat{M}_{4 / 3} \hat{M}_{5 / 6}+\hat{M}_{1} \hat{M}_{7 / 6}\right)
\end{aligned}
$$

\subsection{SWNT nucleation and growth model}

To complete the mathematical model, a reliable model describing the nucleation and growth of SWCNTs from the droplets is needed. Although there exist several models based on molecular dynamics [37, 38] or Monte Carlo [39] approaches, the integration of those models into the previous plasma and particle models is quite challenging because of their large differences in physical scales. For this reason, a simplified model proposed in Refs. $[40,41]$ was adopted in this numerical work. In this model, the nucleation of SWCNTs is initiated as soon as the droplet particles are supersaturated by carbon atoms, and the surplus carbon atoms are assumed to be precipitated out of the surface of the droplets to form SWCNTs. Competing with SWCNT growth is the formation of graphene layers which eventually poison the catalyst.

In order to describe SWCNT nucleation and growth according to the above model, the mean numbers of carbon and metal atoms in a droplet particle (i.e., mean composition of a droplet) should be known. Assuming that the composition of droplets is independent of the droplet size, the mean composition of a droplet at a local position can be calculated by using the following equations:

$$
\begin{gathered}
M_{1}=\int_{0}^{\infty} v_{\mathrm{d}} n\left(v_{\mathrm{d}}\right) \mathrm{d} v_{\mathrm{d}}=\int_{0}^{\infty}\left(v_{\mathrm{d}}^{\mathrm{m}}+v_{\mathrm{d}}^{\mathrm{c}}\right) n\left(v_{\mathrm{d}}\right) \mathrm{d} v_{\mathrm{d}} \\
=\int_{0}^{\infty} v_{\mathrm{d}}^{\mathrm{m}} n\left(v_{\mathrm{d}}\right) \mathrm{d} v_{\mathrm{d}}+\int_{0}^{\infty} v_{\mathrm{d}}^{\mathrm{c}} n\left(v_{\mathrm{d}}\right) \mathrm{d} v_{\mathrm{d}}=M_{1}^{\mathrm{m}}+M_{1}^{\mathrm{c}},
\end{gathered}
$$

and

$$
N_{\mathrm{c}}=\frac{M_{1}^{\mathrm{c}}}{M_{0} \cdot v_{\mathrm{c} 1}}=\frac{\hat{M}_{1}^{\mathrm{c}}}{\hat{M}_{0}} \cdot \frac{v_{\mathrm{m} 1}}{v_{\mathrm{c} 1}}, \quad N_{\mathrm{m}}=\frac{M_{1}^{\mathrm{m}}}{M_{0} \cdot v_{\mathrm{m} 1}}=\frac{\hat{M}_{1}^{\mathrm{m}}}{\hat{M}_{0}} .
$$

$\hat{M}_{1}^{\mathrm{m}}$ and $\hat{M}_{1}^{\mathrm{c}}$ can be calculated by using following transport equations:

$$
\begin{aligned}
\nabla \cdot\left(\rho \boldsymbol{u} \hat{M}_{1}^{\mathrm{m}}\right)=\nabla & \cdot\left[\left(\rho \bar{D}_{\mathrm{d}}+\frac{\mu_{\mathrm{t}}}{S c_{\mathrm{t}}}\right) \nabla \hat{M}_{1}^{\mathrm{m}}\right]-\nabla \cdot\left(\rho \boldsymbol{u}_{\mathrm{th}} \hat{M}_{1}^{\mathrm{m}}\right)+\frac{I}{n_{0}} j \\
& +B_{1}^{\mathrm{m}} \rho\left(S_{\mathrm{m}}-1\right) \hat{M}_{2 / 3}
\end{aligned}
$$

$$
\begin{aligned}
\nabla \cdot\left(\rho \boldsymbol{u} \hat{M}_{1}^{\mathrm{c}}\right)= & \nabla \cdot\left[\left(\rho \bar{D}_{\mathrm{d}}+\frac{\mu_{\mathrm{t}}}{S c_{\mathrm{t}}}\right) \nabla \hat{M}_{1}^{\mathrm{c}}\right]-\nabla \cdot\left(\rho \boldsymbol{u}_{\mathrm{th}} \hat{M}_{1}^{\mathrm{c}}\right)+ \\
& +B_{1}^{\mathrm{c}} \rho\left(S_{\mathrm{c}}-1\right) \hat{M}_{2 / 3} .
\end{aligned}
$$

The saturation limit of carbon atoms in a droplet particle can be obtained by using the relation [42],

$$
N_{\mathrm{c}_{-} \text {sat }}=S_{0} \cdot N_{\mathrm{m}}
$$

The number of surplus carbon atoms is then simply obtained from

$$
N_{\text {c_surp }}=N_{\text {c }}-N_{\text {c_sat }} \text {. }
$$

By utilizing a factor $f_{\text {cnt }}$ (assumed to be 0.5), the number of surplus carbon atoms which will form a SWCNT is given as

$$
N_{\text {cnt }}=f_{\text {cnt }} \cdot N_{\text {c_surp }} \cdot
$$

Finally, the mean length of the SWCNTs can be estimated from the equation,

$$
L_{\mathrm{cnt}}=\left(\frac{m_{\mathrm{c} 1}}{\pi \rho_{\mathrm{g}} \delta_{\mathrm{gph}} d_{\mathrm{cnt}}}\right) N_{\mathrm{cnt}} .
$$

More detailed information on the constants and coefficients employed in the above sub-models is available in the Supporting Information.

\subsection{Computation domain and numerical schemes}

The computational domain considered in the present work is depicted in Fig. 7. The detailed dimension of the processing system is identical to that of the system employed in our previous experimental studies [7]. As shown in Fig. 7, the computational domain mainly consists of a plasma torch zone, a reaction zone, and a quenching zone. Since physical phenomena occurring inside the torch zone are quite different from those occurring inside the reactor zone (i.e., the reaction and quenching zones), the 2-D simulations were carried out in two steps to save computing time. Firstly, the plasma generation and the particle evaporation are simulated without considering the formation of droplets and SWCNTs. Secondly, the formation rates of droplets and SWCNTs inside the reactor zone are calculated using the data extracted at the torch exit, as the boundary condition at the reactor entrance. The operating conditions employed in this numerical study are summarized in Table 2. A mixture of carbon black (CB), nickel, and cobalt was considered as a

and 
feedstock material, with a ratio of $\mathrm{CB} / \mathrm{Ni} / \mathrm{Co}=98.8$ at $\% / 0.6$ at $\% / 0.6$ at $\%$. Details of the particle injection conditions are presented in Table 3.

The density, viscosity, thermal conductivity, specific heat at constant pressure, electrical conductivity, and radiation losses from the plasma gases (i.e., argon and helium) were obtained from the tabulated data [15], which were calculated with the assumption of an LTE. Upon particle evaporation, however, the properties of the plasma could be significantly altered by the presence of the metallic or carbon vapor. Thus, the effects of the particle vapors on the plasma properties - except on the radiational and electrical properties-were taken into account where the detailed data for carbon, nickel, and cobalt vapors were obtained from Refs. [43-44]. To calculate the mixture properties, such as density, thermal conductivity, and viscosity, the mass-weighted mixing law was employed, while the specific heat was calculated using the mixing law. The diffusion coefficients of each species in the mixture were calculated from the modified Chapman equations using Lennard-Jones parameters. Lastly, the thermodynamic properties of the particles injected into the plasma were obtained from $[44,45]$.

To solve the above governing equations, appropriate boundary conditions are required for the computational domain depicted in Fig. 7. The boundary conditions employed in previous thermal plasma modeling were used here for the calculations of thermofluid, electromagnetic fields, species, and the three moments $[26,29,36]$. As a typical example, the boundary conditions used in the plasma generation model is summarized in the Supporting Information.

The 2-D numerical simulations were performed using the commercial code FLUENTC [46]. In this code, the governing equations are transformed into finite difference equations and then solved by the SIMPLE-like pressure correction algorithm proposed by Patankar [47]. The basic modules of FLUENT were employed to solve the conservation

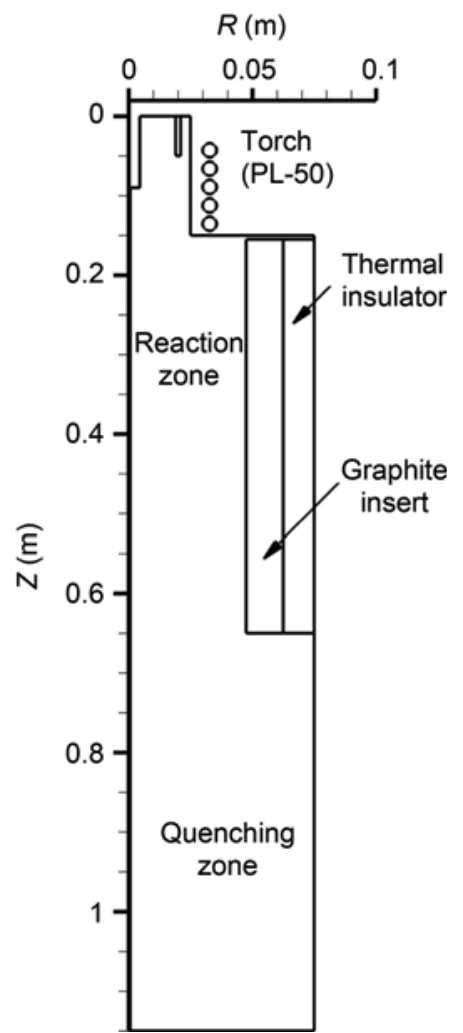

Figure 7 Computational domain employed for the 2-D numerical simulation of the SWCNT synthesis by the induction thermal plasma process

Table 2 Operating condition for the 2-D numerical simulation

\begin{tabular}{ccccccc}
\hline $\begin{array}{c}\text { Net plasma } \\
\text { power }(\mathrm{kW}){ }^{\mathrm{a}}\end{array}$ & $\begin{array}{c}\text { Frequency } \\
(\mathrm{MHz})\end{array}$ & $\begin{array}{c}\text { Pressure } \\
(\mathrm{kPa})\end{array}$ & $\begin{array}{c}\text { Carrier gas } \\
(\mathrm{slpm})\end{array}$ & $\begin{array}{c}\text { Central gas } \\
(\mathrm{slpm})\end{array}$ & $\begin{array}{c}\text { Sheath gas } \\
(\mathrm{slpm})\end{array}$ & $\begin{array}{c}\text { Feed rate } \\
(\mathrm{g} / \mathrm{min})\end{array}$ \\
\hline 28 & 3.0 & 66 & $5(\mathrm{Ar})$ & $25(\mathrm{Ar})$ & $120(\mathrm{He})$ & 1.5 \\
\hline
\end{tabular}

a The conversion efficiency of the RF power supply is assumed to be $70 \%$.

Table 3 Conditions of particle injection for the 2-D numerical simulation

\begin{tabular}{ccccccc}
\hline & $\begin{array}{c}\text { Inlet velocity } \\
(\mathrm{m} / \mathrm{s})\end{array}$ & $\begin{array}{c}\text { Temperature } \\
(\mathrm{K})\end{array}$ & $\begin{array}{c}\text { Mass flow rate } \\
(\mathrm{kg} / \mathrm{s})\end{array}$ & $\begin{array}{c}\text { Max. dia. } \\
(\mathrm{m})\end{array}$ & $\begin{array}{c}\text { Mean dia. } \\
(\mathrm{m})\end{array}$ & $\begin{array}{c}\text { Min. dia. } \\
(\mathrm{m})\end{array}$ \\
\hline Carbon & 11.0 & 300 & $2.4 \times 10^{-5}$ & $3.0 \times 10^{-6}$ & $2.0 \times 10^{-6}$ & $1.0 \times 10^{-6}$ \\
Nickel & 11.0 & 300 & $7.0 \times 10^{-7}$ & $3.0 \times 10^{-6}$ & $2.0 \times 10^{-6}$ & $1.0 \times 10^{-6}$ \\
Cobalt & 11.0 & 300 & $7.0 \times 10^{-7}$ & $3.0 \times 10^{-6}$ & $2.0 \times 10^{-6}$ & $1.0 \times 10^{-6}$ \\
\hline
\end{tabular}


equations for the thermofluid fields, whereas the vector potential equation for the electromagnetic field and the moment transport equations were solved using the user defined functions (UDFs) approach. Once converged solutions were obtained for the thermofluid and moment fields, the growth of SWCNTs was estimated.

\subsection{Numerical results}

Figure 8 shows the distributions of the temperature and species concentration fields (i.e., carbon and nickel vapors) in the entire region of the processing system. As mentioned above, these results were obtained without considering droplet formation. The off-axis profile in the high-temperature zone is clearly observed inside the torch, mainly due to skin depth effect and particle injection through the probe located at the center of the torch. In the reaction zone, the offaxis profile begins to disappear from the entrance of the reactor owing to mixing phenomena, caused by the plasma jet expansion. Owing to the graphite insert, temperatures of over $4000 \mathrm{~K}$ prevail in the entire region of the reaction zone but the temperature decreases rapidly to around $1000 \mathrm{~K}$ in the quenching zone through the intensive heat exchange with the water-cooled reactor walls. The cooling rate at this transition (i.e., $Z=$ $0.65 \mathrm{~m}$ ) was estimated to be around 1.62 $\times 10^{4} \mathrm{~K} / \mathrm{s}$. The broad high-temperature region observed in the reaction zone is highly suitable for the effective growth of SWCNTs. It is clearly seen in Fig. 8(b) that the generation of the vapors is rapid, indicating that induction thermal plasma is ideally suited for the evaporation of solid-phase feedstock materials. To further investigate the particle behaviors in the plasma plume, the trajectories of the each particle injected into the plasma torch were examined and the results are illustrated in Fig. 9. It is obvious from this plot that carbon particles need more time to be evaporated compared to nickel particles and this is mainly due to the higher evaporation temperature of carbon particles.

By taking advantage of these calculations, the nucleation and growth of droplet particles inside the reactor system were simulated and the main results, in particular distributions of droplet number density and droplet diameter, are shown in Fig. 10. Similarly, the temperature and mean droplet size profiles calculated along the reactor axis are presented in Fig. 11(a). The non-monotonic variation in the particle diameter obtained in this work is mainly attributable to the

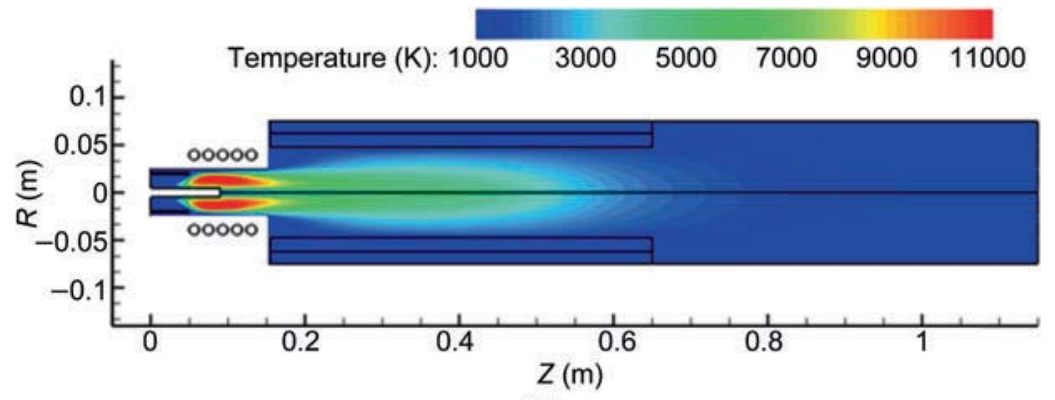

(a)

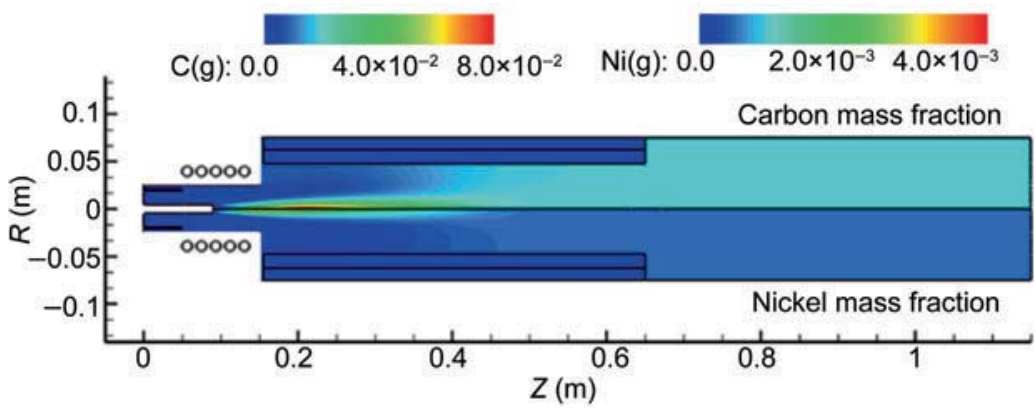

(b)

Figure 8 Calculated distributions of the (a) temperature and species mass fractions inside the induction thermal plasma processing system (plasma power: $28 \mathrm{~kW}$, pressure: $66 \mathrm{kPa}$, plasma gas: mixture of argon and helium, feed rate: $1.5 \mathrm{~g} / \mathrm{min}$, feedstock: CB-Ni-Co/98.8 at\%-0.6 at\%-0.6 at\%)
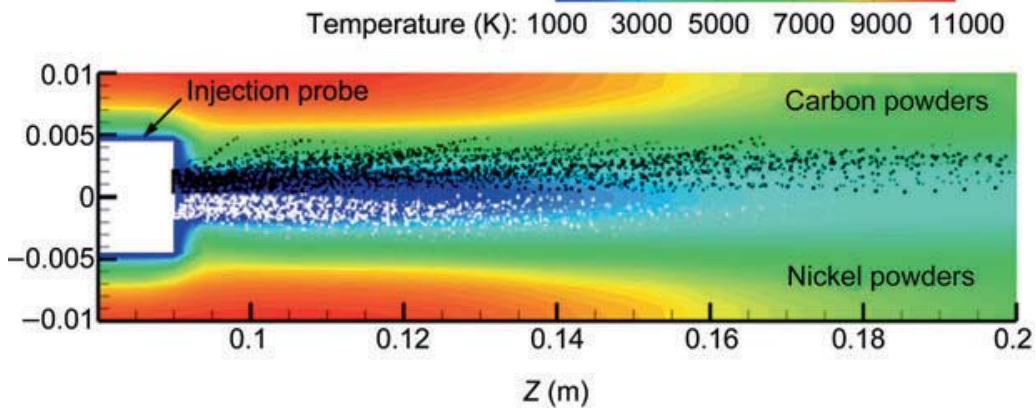

Figure 9 Calculated trajectories of the particles injected into the induction thermal plasma torch (plasma power: $28 \mathrm{~kW}$, pressure: $66 \mathrm{kPa}$, plasma gas: mixture of argon and helium, feed rate: $1.5 \mathrm{~g} / \mathrm{min}$, feedstock: $\mathrm{CB}-\mathrm{Ni}-\mathrm{Co} / 98.8$ at\% -0.6 at\%-0.6 at\%) 
small particles newly generated in the quenching chamber. The mean diameters of the droplets produced range from a few $\mathrm{nm}$ to tens of $\mathrm{nm}$ and are predicted to be around $10 \mathrm{~nm}$ at the exit of the reactor system. To ensure the accuracy of the numerical results, it is important to compare them with the

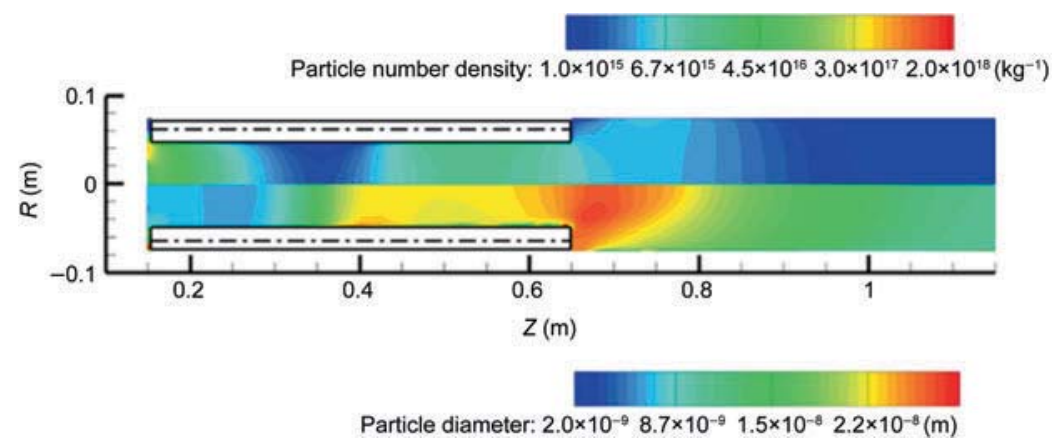

Figure 10 Calculated distributions of the number density of the droplet particles produced (top) and their mean diameter (bottom) (plasma power: $28 \mathrm{~kW}$, pressure: 66 $\mathrm{kPa}$, plasma gas: mixture of argon and helium, feed rate: $1.5 \mathrm{~g} / \mathrm{min}$, feedstock: $\mathrm{CB}-\mathrm{Ni}-$ Co/98.8 at\%-0.6 at\%-0.6 at\%) measured ones. For validation purpose, a TEM image of the SWCNT soot obtained after air oxidation is shown in Fig. 11(b), which shows the size distribution of the metallic nanoparticles present inside the soot. Accounting for the original metal/metal-oxide particle size of 1-2 $\mu \mathrm{m}$, all particles appearing in the TEM image seem to be produced during the synthesis process. The diameter of the particles ranges from $2 \mathrm{~nm}$ to $12 \mathrm{~nm}$ as indicated in Fig. 11(b); therefore, the mean diameter of nanoparticles predicted by the 2-D numerical simulation seems to be in fairly good agreement with the experimental data.

Lastly, the mean length of the SWCNTs produced from a droplet is estimated based on the model described in the previous section. Figure 11(c) shows that the mean length ranges from $1 \mu \mathrm{m}$

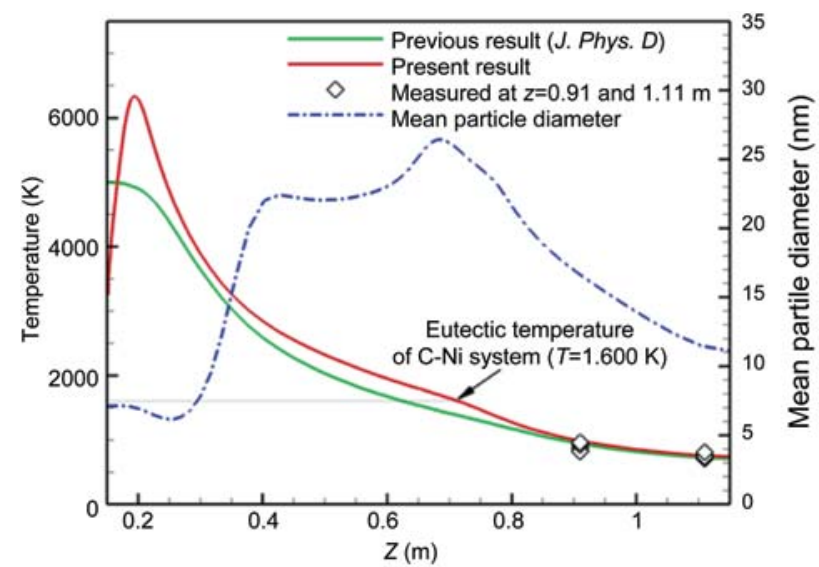

(a)

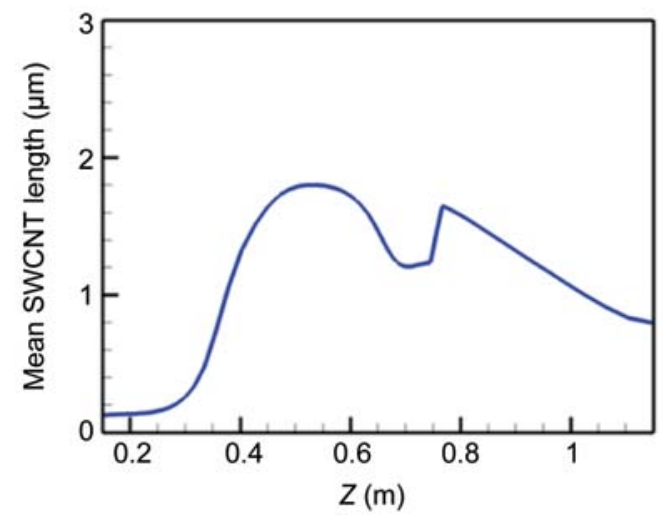

(c)

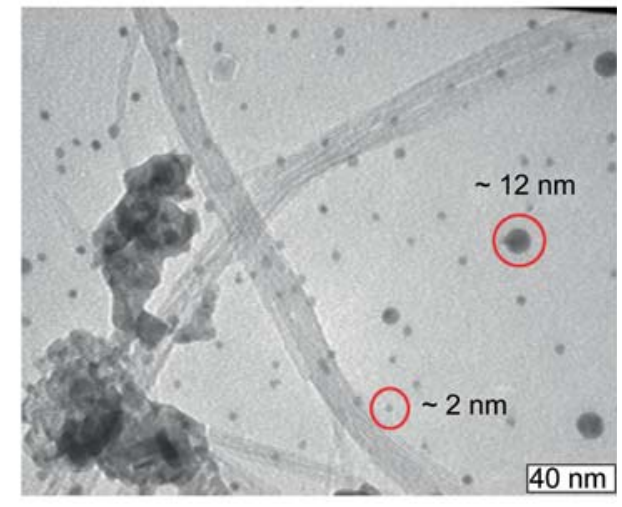

(b)

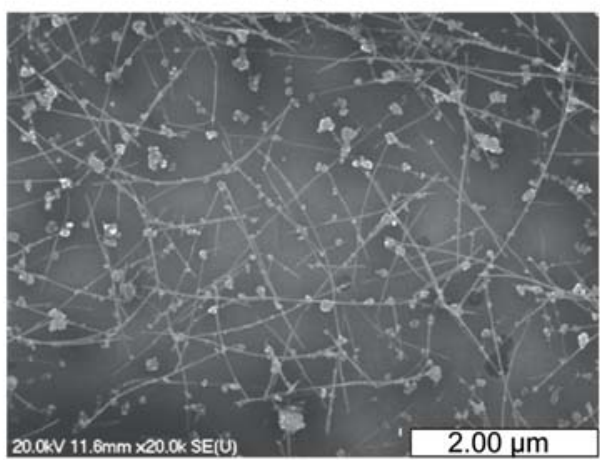

(d)

Figure 11 (a) Axial profiles of the temperature and mean droplet diameter calculated along the axis of the reactor system; (b) TEM image of the SWCNT soot showing the size distribution of the nanoparticles produced; (c) mean length of SWCNTS estimated along the axis of the reactor system; (d) SEM image of the dispersed SWCNTs showing their length distribution 
to $2 \mu \mathrm{m}$. This compares favorably with the values obtained from the SEM images shown in Fig. 11(d). More numerical results on the supersaturation ratio of vapors and the nucleation and growth rates of droplet particles are also available in the Supporting Information.

\section{Concluding remarks}

In the past three years, it has been demonstrated that SWCNTs can be successfully continuously synthesized at a high yield rate of $100 \mathrm{~g} / \mathrm{h}$ in a sheet-like form. Through further optimization studies, the induction thermal plasma process has become a very reliable process, which is now capable of industrial-scale production of SWCNTs. However, there is still a need for further improvement of this process through better understanding of the SWCNT formation mechanism in connection with the plasma conditions established and maintained during the processes. Therefore, the identification and design of the optimum reactor geometry and the related operating parameters, based on detailed studies of the plasma characteristics, will be an important next step of our research.

In this regard, a mathematical model is now being developed and some preliminary results are presented in this article. Comparisons with experimental data demonstrated that the mathematical model developed is reasonable. However, the current model is not completely self-consistent, due to the lack of precise knowledge of some important phenomena and numerical difficulties, thereby imposing many simplifications or assumptions on the model. Especially, nucleation and growth mechanisms of SWCNTs are oversimplified in this model. These phenomena are intrinsically atomic or molecular scale processes (i.e., discrete level) that are affected by macroscopic parameters (e.g., temperature), which are typically describable by using continuum approaches such as fluid dynamics. Therefore, a multi-scale simulation technique should be developed to bridge the gap between the physical phenomena occurring at the different scales. In addition, the droplet formation simulation was conducted without considering the SWCNTs produced on the droplets. Since the various particle processes, such as nucleation, growth, and transport, are strongly influenced by the SWCNTs grown from a droplet, a considerable improvement of this model is needed as well. Lastly, the clustering of carbon atoms and the formation of soot nuclei should be taken into account by using proper mathematical models.

The characterization of the thermal plasma is also very crucial for further validation and subsequent improvement of our current model. Therefore thermal plasma diagnostics, such as enthalpy probe measurement and optical emission spectroscopy (OES), will be carried out in an in situ manner and then more rigorous validation studies will be performed. The scientific information obtained in this work can be utilized for further optimization of the various induction thermal plasma processes developed, not only for the synthesis of SWCNTs but also for the synthesis of other carbon nanostructures, such as fullerenes, carbon-encapsulated nanoparticles, and carbon onions.

\section{Acknowledgements}

This work was supported by the Natural Science and Engineering Research Council (NSERC) of Canada. The authors would also like to acknowledge our collaborators German Cota-Sanchez, Matej Imris, Christopher Kingston, Stéphane Denommée, and Jonathan Genest for their contribution to the present work.

\section{Nomenclature}

$A_{\text {exit }} \quad$ area of reactor exit $\left(\mathrm{m}^{2}\right)$

$A_{\theta} \quad$ tangential component of magnetic vector potential $(\mathrm{T} \cdot \mathrm{m})$

$C_{D}$ drag coefficient

$C_{\mathrm{p}} \quad$ specific heat of fluid at constant pressure $(\mathrm{J} /(\mathrm{kg} \cdot \mathrm{K}))$

$\mathrm{C}_{\mu} \quad$ constant in turbulence model

$C_{1} \quad$ constant in turbulence model

$\mathrm{C}_{2} \quad$ constant in turbulence model

$c_{\mathrm{p}} \quad$ specific heat of particle injected $(\mathrm{J} / \mathrm{kg} \cdot \mathrm{K})$

$D_{\mathrm{d}} \quad$ size dependent diffusion coefficient of droplet produced $\left(\mathrm{m}^{2} / \mathrm{s}\right)$

$\bar{D}_{\mathrm{d}} \quad$ mean diffusion coefficient of droplet produced $\left(\mathrm{m}^{2} / \mathrm{s}\right)$

$D_{k} \quad$ binary diffusion coefficient of species $k\left(\mathrm{~m}^{2} / \mathrm{s}\right)$ 
$d_{\mathrm{cnt}} \quad$ diameter of SWCNT (m)

$d_{\mathrm{d}} \quad$ diameter of droplet produced (m)

$\bar{d}_{\mathrm{d}} \quad$ mean diameter of droplet produced (m)

$d_{\mathrm{p}} \quad$ diameter of particle injected $(\mathrm{m})$

$d_{\mathrm{m} 1} \quad$ diameter of metal monomer $(\mathrm{m})$

$E_{\theta} \quad$ tangential component of electric field $(\mathrm{V} / \mathrm{m})$

$F_{\mathrm{L}} \quad$ Lorentz force $\left(\mathrm{N} / \mathrm{m}^{3}\right)$

$f \quad$ frequency applied to $\mathrm{RF}$ induction coil $(\mathrm{Hz})$

$f_{\text {cnt }} \quad$ percentage of carbon atoms in a droplet which will form an SWCNT

$G$ droplet growth rate by co-condensation $\left(\mathrm{m}^{3} / \mathrm{s}\right)$

$G_{\mathrm{t}} \quad$ product of the turbulent viscosity and viscous dissipation terms $\left(\mathrm{kg} /\left(\mathrm{m} \cdot \mathrm{s}^{3}\right)\right)$

$h \quad$ specific enthalpy of fluid $(\mathrm{J} / \mathrm{kg})$

$h_{\mathrm{c}} \quad$ heat transfer coefficient of fluid $\left(\mathrm{W} /\left(\mathrm{m}^{2} \cdot \mathrm{K}\right)\right)$

$H_{\mathrm{b}} \quad$ latent heat of particle injected at boiling point $(\mathrm{J} / \mathrm{kg})$

$H_{\mathrm{m}} \quad$ latent heat of particle injected at melting point $(\mathrm{J} / \mathrm{kg})$

$H_{\mathrm{r}} \quad$ radial component of magnetic field intensity $(\mathrm{A} / \mathrm{m})$

$H_{z}$ axial component of magnetic field intensity $(\mathrm{A} / \mathrm{m})$

I homogeneous nucleation rate of metal vapor $\left(\mathrm{m}^{-3} \cdot \mathrm{s}^{-1}\right)$

$J_{\text {coil }} \quad$ coil current density $\left(\mathrm{A} / \mathrm{m}^{2}\right)$

$J_{\text {ind }} \quad$ induced current density $\left(\mathrm{A} / \mathrm{m}^{2}\right)$

$j^{*} \quad$ number of monomers contained in a droplet of critical size

$K \quad$ turbulent kinetic energy $\left(\mathrm{m}^{2} / \mathrm{s}^{2}\right)$

$k_{\mathrm{B}} \quad$ Boltzmann constant $(\mathrm{J} / \mathrm{K})$

$L_{\mathrm{cnt}} \quad$ length of SWCNT (m)

$M_{\mathrm{g}} \quad$ molecular weight of gas $(\mathrm{kg} / \mathrm{mol})$

$M_{k} \quad$ moment of $k^{\text {th }}$ order

$\hat{M}_{k} \quad$ moment of $k^{\text {th }}$ order in normalized form

$M_{\mathrm{w}} \quad$ molecular weight of metal $(\mathrm{kg} / \mathrm{mol})$

$\dot{m} \quad$ mass flow rate $(\mathrm{kg} / \mathrm{s})$

$m_{\mathrm{c} 1} \quad$ mass of carbon monomer $(\mathrm{kg})$

$m_{\mathrm{m} 1} \quad$ mass of metal monomer $(\mathrm{kg})$

$m_{\mathrm{p}} \quad$ mass of particle injected $(\mathrm{kg})$

$N \quad$ total number of droplet produced $\left(\mathrm{m}^{-3}\right)$

$N_{\mathrm{A}} \quad$ Avogadro constant $\left(\mathrm{mol}^{-1}\right)$

$N_{\mathrm{c}} \quad$ mean number of carbon atoms in a droplet produced

$N_{\text {cnt }} \quad$ number of surplus carbon atoms in a droplet which will form an SWCNT

$N_{\text {c.sat }}$ maximum number of carbon atoms in a droplet produced (saturation limit of carbon atoms)

$N_{\text {c_surp }}$ number of surplus carbon atoms in a droplet produced

$N_{\mathrm{m}} \quad$ mean number of metal atoms in a droplet produced

$N_{\mathrm{ml}}$ number of surplus carbon atoms in a droplet which will form a graphene monolayer

$\mathrm{Nu} \quad$ Nusselt number

$n_{0} \quad$ reference monomer concentration employed for normalization of moments $\left(\mathrm{m}^{-3}\right)$

$n_{\mathrm{ms}} \quad$ metal vapor concentration at saturation $\left(\mathrm{m}^{-3}\right)$

$n_{\mathrm{cs}} \quad$ carbon vapor concentration at saturation $\left(\mathrm{m}^{-3}\right)$

$P_{\text {ohm }}$ heat generation by ohmic heating $\left(\mathrm{W} / \mathrm{m}^{3}\right)$

$P_{\mathrm{s}}^{\mathrm{c}} \quad$ saturation pressure of carbon $(\mathrm{Pa})$

$P_{\mathrm{s}}^{\mathrm{m}} \quad$ saturation pressure of metal $(\mathrm{Pa})$

$P_{\text {vap }}^{\mathrm{c}} \quad$ vapor pressure of carbon $(\mathrm{Pa})$

$P_{\text {vap }}^{\mathrm{m}} \quad$ vapor pressure of metal $(\mathrm{Pa})$

$p$

static pressure of fluid $(\mathrm{Pa})$

$\mathrm{Pr} \quad$ Prandtl number of fluid

$\mathrm{Pr}_{\varepsilon} \quad$ constant in turbulence model

$\mathrm{Pr}_{\mathrm{K}} \quad$ constant in turbulence model

$P r_{\mathrm{t}} \quad$ turbulent Prandtl number

$R \quad$ universal gas constant $(\mathrm{J} /(\mathrm{mol} \cdot \mathrm{K}))$

$R e_{\mathrm{p}} \quad$ particle Reynolds number

$R_{\text {rad }} \quad$ radiational loss taken into account by using net emission coefficient $\left(\mathrm{W} / \mathrm{m}^{3}\right)$

$S_{\mathrm{c}} \quad$ supersaturation ratio of carbon vapor

$S c_{\mathrm{t}} \quad$ turbulent Schmidt number

$S_{\mathrm{m}} \quad$ supersaturation ratio of metal vapor

$S_{\mathrm{p}}^{\mathrm{c}} \quad$ mass generation through evaporation of particle injected $\left(\mathrm{kg} /\left(\mathrm{m}^{3} \cdot \mathrm{s}\right)\right)$

$S_{\mathrm{p}}^{\mathrm{e}} \quad$ heat exchange with particle injected $\left(\mathrm{W} / \mathrm{m}^{3}\right)$

$S_{\mathrm{p}}^{\mathrm{k}} \quad$ mass generation of $k^{\text {th }}$ species through evaporation of particle injected $\left(\mathrm{kg} /\left(\mathrm{m}^{3} \cdot \mathrm{s}\right)\right)$

$S_{\mathrm{p}}^{\mathrm{m}} \quad$ momentum exchange with particle injected $\left(\mathrm{N} / \mathrm{m}^{3}\right)$

$S_{0} \quad$ bulk solubility of carbon in metal at melting temperature

$s_{\mathrm{m} 1} \quad$ surface area of metal monomer $\left(\mathrm{m}^{2}\right)$

$T$ temperature of fluid (K)

$T_{\mathrm{a}} \quad$ temperature of ambient gas (K)

$T_{\mathrm{b}} \quad$ boiling temperature of particle injected (K)

$T_{\mathrm{m}} \quad$ melting temperature of particle injected (K)

$T_{\mathrm{p}} \quad$ temperature of particle injected (K)

$u \quad$ velocity of fluid $(\mathrm{m} / \mathrm{s})$

$u_{\mathrm{p}} \quad$ velocity of particle injected $(\mathrm{m} / \mathrm{s})$

$\boldsymbol{u}_{\text {th }} \quad$ thermophoretic velocity $(\mathrm{m} / \mathrm{s})$

$u \quad$ axial velocity of fluid $(\mathrm{m} / \mathrm{s})$

$v$ radial velocity of fluid $(\mathrm{m} / \mathrm{s})$

$w \quad$ swirl velocity of fluid $(\mathrm{m} / \mathrm{s})$

$x \quad$ fraction of liquid phase in particle

$W_{k} \quad$ molecular weight of species $k(\mathrm{~kg} / \mathrm{mol})$

$Y_{k} \quad$ mass fraction of species $k$ 


\section{Greek letters}

$\delta \quad$ Dirac-delta function

$\delta_{\text {gph }} \quad$ thickness of graphene layer (m)

$\varepsilon \quad$ dissipation rate of turbulent kinetic energy $\left(\mathrm{m}^{2} / \mathrm{s}^{3}\right)$

$\varepsilon_{\mathrm{p}} \quad$ emissivity of particle

$\Theta \quad$ dimensionless surface tension

$x$ laminar thermal conductivity of fluid $(\mathrm{W} /(\mathrm{m} \cdot \mathrm{K}))$

$\varkappa_{\text {eff }} \quad$ effective thermal conductivity of fluid $(\mathrm{W} /(\mathrm{m} \cdot \mathrm{K}))$

$x_{\mathrm{t}} \quad$ turbulent thermal conductivity of fluid $(\mathrm{W} /(\mathrm{m} \cdot \mathrm{K}))$

$\lambda \quad$ mean free path $(\mathrm{m})$

$\mu \quad$ laminar viscosity of fluid $(\mathrm{kg} /(\mathrm{m} \cdot \mathrm{s}))$

$\mu_{\text {eff }} \quad$ effective viscosity of fluid $(\mathrm{W} /(\mathrm{m} \cdot \mathrm{s}))$

$\mu_{\mathrm{t}} \quad$ turbulent viscosity of fluid $(\mathrm{kg} /(\mathrm{m} \cdot \mathrm{s}))$

$\mu_{0} \quad$ magnetic permeability of free space $\left(\mathrm{N} / \mathrm{A}^{2}\right)$

$v_{\mathrm{c} 1} \quad$ monomer volume of carbon $\left(\mathrm{m}^{3}\right)$

$v_{\mathrm{d}} \quad$ volume of droplet produced $\left(\mathrm{m}^{3}\right)$

$v_{\mathrm{d}}^{\mathrm{c}} \quad$ volume of carbon in a droplet produced $\left(\mathrm{m}^{3}\right)$

$v_{\mathrm{d}}^{\mathrm{m}} \quad$ volume of metal in a droplet produced $\left(\mathrm{m}^{3}\right)$

$v_{\mathrm{d}}^{*} \quad$ critical volume of droplet $\left(\mathrm{m}^{3}\right)$

$v_{\mathrm{g}} \quad$ geometric mean volume of droplet produced $\left(\mathrm{m}^{3}\right)$

$v_{\mathrm{m} 1} \quad$ monomer volume of metal $\left(\mathrm{m}^{3}\right)$

$\xi$ droplet surface availability for carbon atom condensation

$\rho \quad$ mass density of fluid $\left(\mathrm{kg} / \mathrm{m}^{3}\right)$

$\rho_{\mathrm{d}} \quad$ mass density of droplet produced $\left(\mathrm{kg} / \mathrm{m}^{3}\right)$

$\rho_{\mathrm{g}} \quad$ mass density of graphitic layer $\left(\mathrm{kg} / \mathrm{m}^{3}\right)$

$\rho_{\mathrm{m}} \quad$ mass density of metal $\left(\mathrm{kg} / \mathrm{m}^{3}\right)$

$\rho_{\mathrm{p}} \quad$ mass density of particle injected $\left(\mathrm{kg} / \mathrm{m}^{3}\right)$

$\sigma \quad$ electrical conductivity $\left(\Omega^{-1} \cdot \mathrm{m}^{-1}\right)$

$\sigma_{\mathrm{g}} \quad$ geometric standard deviation of droplet size distribution

$\sigma_{\mathrm{sb}} \quad$ Stefan-Boltzmann constant $\left(\mathrm{W} /\left(\mathrm{m}^{2} \cdot \mathrm{K}^{4}\right)\right)$

$\sigma_{\text {st }} \quad$ surface tension $(\mathrm{N} / \mathrm{m})$

$\tau \quad$ viscous stress tensor $\left(\mathrm{N} / \mathrm{m}^{2}\right)$

Electronic Supplementary Material: Supplementary material is available in the online version of this article at http://dx.doi.org/10.1007/s12274-009-9085-9 and accessible free of charge.

\section{References}

[1] Saito, R.; Dresselhaus, G.; Dresselhaus, M. S. Physical Properties of Carbon Nanotubes; Imperial College Press: London, 1998.

[2] Hayamizu, Y.; Yamada, T.; Mizuno, K.; Davis, R. C.; Futaba, D. N.; Yumura, M.; Hata, K. Integrated three- dimensional microelectromechanical devices from processable carbon nanotube wafers. Nat. Nanotechnol. 2008, 3, 289-294.

[3] Liu, Z.; Tabakman, S.; Welsher, K.; Dai, H. Carbon nanotubes in biology and medicine: In vitro and in vivo detection, imaging and drug delivery. Nano Res. 2009, 2, 85-120.

[4] Ci, L.; Suhr, J.; Pushparaj, V.; Zhang, X.; Ajayan, P. M. Continuous carbon nanotube reinforced composites. Nano Lett. 2008, 8, 2762-2766.

[5] Liu, C.; Cheng, H. M. Carbon nanotubes for clean energy applications. J. Phys. D: Appl. Phys. 2005, 38, R231-R252.

[6] Mauter, M. S.; Elimelech, M. Environmental applications of carbon-based nanomaterials. Environ. Sci. Technol. 2008, 42, 5843-5859.

[7] Kim, K. S.; Cota-Sanchez, G.; Kingston, C. T.; Imris, M.; Simard, B.; Soucy, G. Large-scale production of single-walled carbon nanotubes by induction thermal plasma. J. Phys. D: Appl. Phys. 2007, 40, 2375-2387.

[8] Ostrikov, K.; Murphy, A. B. Plasma-aided nanofabrication: Where is the cutting edge? J. Phys. D: Appl. Phys. 2007, 40, 2223-2241.

[9] Gonzalez-Aguilar, J.; Moreno, M.; Fulcheri, L. Carbon nanostructures production by gas-phase plasma processes at atmospheric pressure. J. Phys. D: Appl. Phys. 2007, 40, 2361-2374.

[10] Boulos, M. I. Thermal plasma processing. IEEE Trans. Plasma Sci. 1991, 19, 1078-1089.

[11] Boulos, M. I. The inductively coupled R.F. (radio frequency) plasma. Pure Appl. Chem. 1985, 57, 13211352.

[12] Boulos, M. I. The inductively coupled radio frequency plasma. High Temp. Mater. Proc. 1997, 1, 17-39.

[13] Watanabe, T.; Notoya, T.; Ishigaki, T.; Kuwano, H.; Tanaka, H.; Moriyoshi, Y. Growth mechanism for carbon nanotubes in a plasma evaporation process. Thin Solid Films 2006, 506, 263-267.

[14] Guo, T.; Nikolaev, P.; Thess, A.; Colbert, D. T.; Smalley, R. E. Catalytic growth of single-walled nanotubes by laser vaporization. Chem. Phys. Lett. 1995, 243, 49-54.

[15] Boulos, M. I.; Fauchais, P.; Pfender, E. Thermal Plasmas, Fundamentals and Applications; Plenum: New York, 1994.

[16] Lange, H.; Bystrzejewski, M.; Huczko, A. Influence of carbon structure on carbon nanotube formation and carbon arc plasma. Diamond Relat. Mater. 2006, 15, 1113 -1116 .

[17] Laplaze, D.; Alvarez, L.; Guillard, T.; Badie, J. M.; 
Flamant, G. Carbon nanotubes: Dynamics of synthesis processes. Carbon 2002, 40, 1621-1634.

[18] Kim, K. S.; Imris, M.; Shahverdi, A.; Alinejad, Y.; Soucy, G. Single-walled carbon nanotubes prepared by largescale induction thermal plasma process: Synthesis, characterization, and purification. J. Phys. Chem. C 2009, 113, 4340-4348.

[19] Bachilo, S. M.; Strano, M. S.; Kittrell, C.; Hauge, R. H.; Smalley, R. E.; Weisman, R. B. Structure-assigned optical spectra of single-walled carbon nanotubes. Science 2002, 304, 2361-2366.

[20] Eswaramoorthy, M.; Sen, R.; Rao, C. N. R. A study of micropores in single-walled carbon nanotubes by the adsorption of gases and vapors. Chem. Phys. Lett. 1999, 304, 207-210.

[21] Farhat, S.; Scott, C. D. Review of the arc process modeling for fullerene and nanotube production. $J$. Nanosci. Nanotechnol. 2006, 6, 1189-1210.

[22] Brown, D. P.; Nasibulin, A. G.; Kauppinen, E. I. CFDaerosol modeling of the effects of wall composition and inlet conditions on carbon nanotube catalyst particle activity. J. Nanosci. Nanotechnol. 2008, 8, 3803-3819.

[23] Moradian, A.; Mostaghimi, J.; Kim, K. S.; Soucy, G. Modeling large-scale synthesis of single-walled carbon nanotubes by induction thermal plasma. High Temp. Mater. Proc., in press.

[24] Saito, Y. Nanoparticles and filled nanocapsules. Carbon 1995, 33, 979-988.

[25] Mostaghimi, J.; Boulos, M. I. Two-dimensional electromagnetic-field effects in induction plasma modeling. Plasma Chem. Plasma Process. 1989, 9, 25-44.

[26] Xue, S. W.; Proulx, P.; Boulos, M. I. Extended-field electromagnetic model for inductively coupled plasma. J. Phys. D: Appl. Phys. 2001, 34, 1897-1906.

[27] Proulx, P.; Mostaghimi, J.; Boulos, M. I. Heating of powders in an RF inductively coupled plasma under dense loading conditions. Plasma Chem. Plasma Process. 1987, 7, 29-52.

[28] Chen, X. Particle heating in a thermal plasma. Pure Appl. Chem. 1988, 60, 651-662.

[29] Shigeta, M.; Watanabe, T. Numerical investigation of cooling effect on platinum nanoparticle formation in inductively coupled thermal plasmas. J. Appl. Phys. 2008, 103, 074903.

[30] Boulos, M. I. Heating of powders in the fire ball of an induction plasma. IEEE Trans. Plasma Sci. 1978, 6, 93106.

[31] Ranz, W. E.; Marshall, W. R. Evaporation from drops,
Part I. Chem. Eng. Prog. 1952, 48, 141-146.

[32] Ranz, W. E.; Marshall, W. R. Evaporation from drops, Part II. Chem. Eng. Prog. 1952, 48, 173-180.

[33] Friedlander, S. K. Smoke, Dust and Haze, Fundamental of Aerosol Dynamics; Oxford University Press: New York, 2000.

[34] Phanse, G. M.; Pratsinis, S. E. Theory for aerosol generation in laminar-flow condensers. Aerosol Sci. Technol. 1989, 11, 100-119.

[35] Girshick, S. L.; Chiu, C. P.; Mcmurry, P. H. Timedependent aerosol models and homogeneous nucleation rates. Aerosol Sci. Technol. 1990, 13, 465-477.

[36] Bilodeau, J. F.; Proulx, P. A mathematical model for ultrafine iron powder growth in a thermal plasma. Aerosol Sci. Technol. 1996, 24, 175-189.

[37] Ding, F.; Bolton, K.; Rosen, A. Nucleation and growth of single-walled carbon nanotubes: A molecular dynamics study. J. Phys. Chem. B 2004, 108, 1736917377.

[38] Ding, F.; Rosen, A.; Bolton, K. Molecular dynamics study of the catalyst particle size dependence on carbon nanotube growth. J. Chem. Phys. 2004, 121, 2775-2779.

[39] Roland, C.; Bernholc, J.; Brabec, C.; Nardelli, M. B.; Maiti, A. Theoretical investigations of carbon nanotube growth. Mol. Simulat. 2000, 25, 1-12.

[40] Celnik, M.; West, R.; Morgan, N.; Kraft, M.; Moisala, A.; Wen, J.; Green, W.; Richter, H. Modelling gas-phase synthesis of single-walled carbon nanotubes on iron catalyst particles. Carbon 2008, 46, 422-433.

[41] Wen, J. Z.; Celnik, M.; Richter, H.; Treska, M.; Sande, J. B. V.; Kraft, M. Modelling study of single walled carbon nanotube formation in a premixed flame. J. Mater. Chem. 2008, 18, 1582-1591.

[42] Moisala, A.; Nasibulin, A. G.; Kauppinen, E. I. The role of metal nanoparticles in the catalytic production of single-walled carbon nanotubes - A review. J. Phys. Condens. Matter 2003, 15, S3011-S3035.

[43] Pousse, J.; Chervy, B.; Bilodeau, J. F.; Gleizes, A. Thermodynamic and transport properties of argon/ carbon and helium/carbon mixtures in fullerene synthesis. Plasma Chem. Plasma Process. 1996, 16, 605-634.

[44] Gale, W. F.; Totemeier, T. C. Smithells Metals Reference Book. Elsevier Butterworth-Heinemann: Oxford, 2004.

[45] Nesmeyanov, A. N. Vapor Pressure of the Chemical Elements; Elsevier: New York, 1963.

[46] FLUENT User's Guide. Version 6.2, 2005.

[47] Patankar, S. V. Numerical Fluid Flow and Heat Transfer; Hemisphere: New York, 1980. 